\title{
Observation of a VHE cosmic-ray flare-signal with the L3+C muon spectrometer
}

O. Adriani ${ }^{\text {i }}$, M. van den Akker ${ }^{\text {o }}$, T. Aziz ${ }^{\text {h }}$, J. Bähr ${ }^{\mathrm{r}}$, S. Banerjee ${ }^{\text {h }}$, F. Becattini ${ }^{\mathrm{i}}$, L. Bellucci ${ }^{\mathrm{i}}$, B.L. Betev ${ }^{\text {s, J.J. Blaising }}{ }^{\mathrm{d}, \mathrm{j}}$, G.J. Bobbink ${ }^{\mathrm{b}}$, S. Bottai ${ }^{\mathrm{i}}$, D. Bourilkov ${ }^{\mathrm{s}}$, A. Cartacci $^{\mathrm{i}}$, M. Chemarin ${ }^{\mathrm{m}}$, G. Chen ${ }^{\mathrm{e}}$, G.M. Chen ${ }^{\mathrm{e}}$, H.S. Chen ${ }^{\mathrm{e}}$, T. Chiarusi ${ }^{\mathrm{i}}$, G. Coignet ${ }^{\mathrm{d}}$, L.K. Ding ${ }^{\mathrm{e}}$, I. Duran ${ }^{\mathrm{q}}$, A. Eline ${ }^{\mathrm{j}}$, H. El Mamouni ${ }^{\mathrm{m}}$, G. Faber ${ }^{\mathrm{s}}$, J. Fay ${ }^{\mathrm{m}}$, F. Filthaut ${ }^{\mathrm{o}}$, S.N. Ganguli ${ }^{\mathrm{h}}$, Z.F. Gong ${ }^{1}$, H.J. Grabosch ${ }^{\mathrm{r}}$,

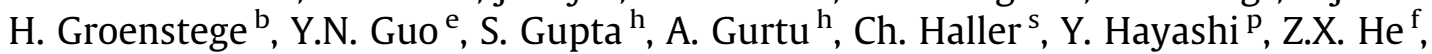
T. Hebbeker ${ }^{a}$, A. Hervé ${ }^{j}$, H. Hofer ${ }^{s}$, H. Hofer Jr. ${ }^{s}$, A.X. Huo ${ }^{e}$, N. Ito ${ }^{p}$, C.L. Jing ${ }^{e}$, L.W. Jones ${ }^{c}$, V. Kantserov ${ }^{\text {r,1 }}$, S. Kawakami ${ }^{\text {, }}$, W. Kittel ${ }^{\circ}$, A.C. König ${ }^{\circ}$, E. Kok ${ }^{\text {b }}$, H.H. Kuang ${ }^{\text {e }}$, J. Kuijpers ${ }^{\circ}$, P. Ladron de Guevara ${ }^{n}$, P. Le Coultre ${ }^{\mathrm{s}, *}$, Y. Lei $^{\mathrm{e}}$, H. Leich $^{\mathrm{r}}$, R. Leiste ${ }^{\mathrm{r}}$, L. Li ${ }^{\mathrm{e}}$, Z.C. Li $^{\mathrm{e}}$, Z.A. Liu ${ }^{\mathrm{e}}$, W. Lohmann ${ }^{\mathrm{r}}$, Y.S. Lu ${ }^{\mathrm{e}}$, W.G. Ma ${ }^{1}$, X.H. Ma ${ }^{\mathrm{e}}$, Y.Q. Ma ${ }^{\mathrm{e}}$, S. Mele ${ }^{\mathrm{j}}$, X.W. Meng ${ }^{\mathrm{e}}$, M. Meschini ${ }^{\mathrm{i}}$, W.J. Metzger ${ }^{\mathrm{o}}$, A. van Mil ${ }^{\circ}$, H. Milcent ${ }^{\mathrm{j}}$, G.B. Mohanty ${ }^{\mathrm{h}}$, B. Monteleoni ${ }^{\mathrm{i}, 2}$, R. Nahnhauer $^{\mathrm{r}}$, V.A. Naumov ${ }^{\mathrm{i}}$, H. Nowak $^{\mathrm{r}}$, J.-F. Parriaud ${ }^{\mathrm{m}}$, F. Pauss ${ }^{\mathrm{s}}$, B. Petersen ${ }^{\mathrm{o}}$, M. Pieri ${ }^{\mathrm{i}}$, M. Pohl ${ }^{\mathrm{k}}$, V. Pojidaev ${ }^{\mathrm{i}}$, C.R. Qing ${ }^{\mathrm{f}}$, R. Ramelli ${ }^{\mathrm{s}}$, R. Ranieri ${ }^{i}$, K.C. Ravindran ${ }^{\mathrm{h}}$, P. Rewiersma ${ }^{\mathrm{b}, 2}$, S. Riemann ${ }^{\mathrm{r}}$, A. Rojkov ${ }^{\mathrm{i}, \mathrm{o}, \mathrm{s}}$, L. Romero ${ }^{\mathrm{n}}$, V. Schmitt ${ }^{\mathrm{g}}$, B. Schoeneich ${ }^{r}$, D.J. Schotanus ${ }^{\text {o }}$, C.Q. Shen ${ }^{\mathrm{e}}$, P. Spillantini ${ }^{\mathrm{i}}$, H. Sulanke ${ }^{\mathrm{r}}$, X.W. Tang ${ }^{\mathrm{e}}$, C. Timmermans ${ }^{\mathrm{o}}$, S.C. Tonwar ${ }^{\mathrm{h}}$, G. Trowitzsch ${ }^{\mathrm{r}}$, M. Unger ${ }^{\mathrm{r}}$, H. Verkooijen ${ }^{\mathrm{b}}$, R.T. Van de Walle ${ }^{\mathrm{o}}$, H. Vogt $^{\mathrm{r}}$, R.G. Wang ${ }^{\mathrm{e}}$, Q. Wang ${ }^{\circ}$, X.L. Wang ${ }^{1}$, X.W. Wang ${ }^{\mathrm{e}}$, Z.M. Wang ${ }^{1}$, R. van Wijk ${ }^{\mathrm{b}}$, T.A.M. Wijnen ${ }^{\mathrm{o}}$, H. Wilkens ${ }^{\mathrm{o}}$,

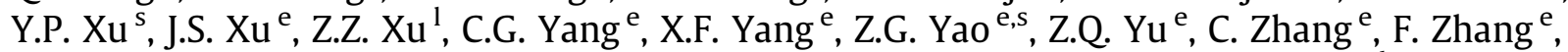
J. Zhang ${ }^{\text {e, S. Zhang }}{ }^{\mathrm{e}}$, S.J. Zhou ${ }^{\mathrm{e}}$, G.Y. Zhu ${ }^{\mathrm{e}}$, Q.Q. Zhu ${ }^{\mathrm{e}}$, H.L. Zhuang ${ }^{\mathrm{e}}$, A.N.M. Zwart ${ }^{\mathrm{b}}$, The L3+C Collaboration

a III. Physikalisches Institut, RWTH, D-52056 Aachen, Germany ${ }^{3}$

${ }^{\mathrm{b}}$ NIKHEF, and University of Amsterdam, NL-1009 DB Amsterdam, The Netherlands

${ }^{\mathrm{C}}$ University of Michigan, Ann Arbor, MI 48109, USA

${ }^{\mathrm{d}}$ LAPP, IN2P3-CNRS, BP 110, F-74941 Annecy-le-Vieux CEDEX, France

${ }^{\mathrm{e}}$ Institute of High Energy Physics, IHEP, 100039 Beijing, China ${ }^{4}$

${ }^{\mathrm{f}}$ ITP, Academia Sinica, 100039 Beijing, China

${ }^{\mathrm{g}}$ Humboldt University, D-10115 Berlin, Germany

${ }^{\text {h }}$ Tata Institute of Fundamental Research, Mumbai (Bombay) 400 005, India

${ }^{\mathrm{i}}$ University of Florence and INFN, Sezione di Firenze, I-50019 Sesto Fiorentino, Italy

${ }^{\mathrm{j}}$ European Laboratory for Particle Physics, CERN, CH-1211 Geneva 23, Switzerland

${ }^{\mathrm{k}}$ University of Geneva, CH-1211 Geneva 4, Switzerland

${ }^{1}$ Chinese University of Science and Technology, USTC, Hefei Anhui 230 029, China ${ }^{4}$

${ }^{\mathrm{m}}$ Institut de Physique Nucléaire de Lyon, IN2P3-CNRS, Université Claude Bernard, F-69622 Villeurbanne, France

${ }^{\mathrm{n}}$ Centro de Investigaciones Energéticas, Medioambientales y Tecnológicas, CIEMAT, E-28040 Madrid, Spain ${ }^{5}$

${ }^{\circ}$ Radboud University and NIKHEF, NL-6525 ED Nijmegen, The Netherlands

${ }^{\mathrm{P}}$ Osaka City University, Osaka 558-8585, Japan

${ }^{\mathrm{q}}$ University of Santiago de Compostela, E-15706 Santiago, Spain

${ }^{\mathrm{r}}$ DESY, D-15738 Zeuthen, Germany

${ }^{\mathrm{s}}$ Eidgenössische Technische Hochschule, ETH Zürich, CH-8093 Zürich, Switzerland

\footnotetext{
* Corresponding author. Tel.: +4122 767 2402; fax: +41 227671520.

E-mail address: Pierre.Le.Coultre@cern.ch (P. Le Coultre).

1 On leave from the Moscow Physical Engineering Institute (MePhl).

2 Deceased.

3 Supported by the German Bundesministerium für Bildung, Wissenschaft, Forschung und Technologie.

${ }^{4}$ Supported by the National Natural Science Foundation of China.

${ }^{5}$ Supported also by the Comisión Interministerial de Ciencia y Tecnología.
} 


\section{A R T I C L E I N F O}

\section{Article history:}

Received 3 February 2009

Received in revised form 3 August 2009

Accepted 4 November 2009

Available online 17 November 2009

Keywords:

Gamma source

Muons

$\mathrm{L} 3+\mathrm{C}$ spectrometer

\section{A B S T R A C T}

The data collected by the L3+C muon spectrometer at the CERN Large Electron-Positron collider, LEP, have been used to search for short duration signals emitted by cosmic point sources. A sky survey performed from July to November 1999 and from April to November 2000 has revealed one single flux enhancement (chance probability $=2.6 \times 10^{-3}$ ) between the 17th and 20th of August 2000 from a direction with a galactic longitude of $(265.02 \pm 0.42)^{\circ}$ and latitude of $(55.58 \pm 0.24)^{\circ}$. The energy of the detected muons was above $15 \mathrm{GeV}$.

(C) 2009 Elsevier B.V. All rights reserved.

\section{Introduction}

Today some 70 sources are known emitting $\mathrm{TeV}$ gamma-rays and even more providing signals above $100 \mathrm{GeV}$ [1-3]. Galactic ones have been identified as Super-Novae Remnants (SNR), Pulsars (PSR) and Plerions (PWN), Binaries (B) [4], or regions of shocked stellar winds. Extragalactic sources are Active Galactic Nuclei (AGN), Blazars (BL Lacs), ejecting jets pointing towards the Earth, and, as more recently observed, a Starburst-Galaxy [5], a RadioGalaxy (the jet not pointing to the Earth) [6] and a "dark source" (without emission of radio-, or X-ray signals) [7]. Some of theses sources have first been discovered by EGRET [8] and other satellite experiments (e.g. [9]) recording $\mathrm{MeV}$ to $\mathrm{GeV}$ gamma rays. Ground based Cherenkov telescopes [10-17] and in rarer cases air shower scintillator arrays [18-20], have become TeV gamma ray detectors. Signals of up to $80 \mathrm{TeV}$ have been recorded, e.g. as from the Crab [21], or from SNR RXJ1713.7-3946 [22].

The measured high-energy gamma spectra are unfortunately incomplete in the sense that up to quite recently there is a lack of data in the energy range between $\sim 10$ and $300 \mathrm{GeV}$, and a cut-off at energies above typically $5-10 \mathrm{TeV}$. The latter is due to the absorption of the gamma rays by interactions with the infrared or background radiation over longer distances [23,24], or due to absorption in the neighbourhood of the acceleration region. This masks the direct observation of the full energy spectrum. Another characteristic of these sources is that the signal can be of steady, periodic, and/or sporadic nature. These facts indicate the problems to be met while trying to determine the mechanism(s) at work for accelerating particles to high energies and converting finally the energy into the observed gamma fluency.

To test models one has first to find new sources in order to increase the statistics of particular data for the different kind of sources, and secondly to build more sensitive detectors, capable of measuring the spectra also in the energy range between 10 and $300 \mathrm{GeV}$. Such efforts have been undertaken in the last few years [25-30] and new results are already published about sources emitting primary gamma rays with energies above $\approx 50-200 \mathrm{GeV}$. While imaging Cherenkov detectors can detect only one source at a time with relatively good angular and energy resolution, sky survey air shower experiments have the opportunity to continuously search large sky regions for new signals, although with slightly less good angular and energy resolution, as well as higher energy thresholds (e.g. ARGO [31], TIBET-AS $\gamma$ [19], GRAPES-III [32]). The MILAGRO air shower Cherenkov-experiment [33] offers a continuous sky survey capability for relatively low primary gamma energies around a few TeV [34].

In this paper a search for point sources is presented based on the detection of secondary muons $[35,36]$. High energy muons originating from the decay of photo-produced pions or $K$-mesons have practically the same direction as the incoming gamma ray and point also back to the source. The effective pointing uncertainty is below $0.1^{\circ}$ above $20 \mathrm{GeV}$ (see below). The detection probability is reduced compared to the detection of the electromagnetic component, due to the much smaller cross-section for photo-production compared to the electron-pair production cross-section $[37,38]$ Nevertheless, if both, the flux and the energy of the signal are large enough, a source can be found. The original idea of the $\mathrm{L} 3+\mathrm{C}$ collaboration was not to detect sources emitting steady signals, since the energy flow of all known sources is below the detection threshold of the $\mathrm{L} 3+\mathrm{C}$ detector, but to record possible intense, very high energy flare-signals: recently exceptional bursts have been observed by the MAGIC collaboration from Mkn 501 [39]. The HESS collaboration observed signals originating from the BL Lac object (PKS 2155-304) [40] and from the AGN radio galaxy M87 with flux doubling times of down to a few days [6].

Deep underground detectors, like e.g. MACRO [41] and NUSEX [42] have also been used for this purpose. But probably due to the high muon energy threshold, MACRO has not recorded any signal in a long lasting sky survey [43]. The SOUDAN collaboration, on the contrary, has observed a signal from Cyg X-3 in 1985 and a second signal in 1991 [44]. Despite of the coincidence with a strong radio burst, this has never been confirmed by another experiment [45]. Low energy muons (crossing typically one hadronic interaction length of absorber) have been recorded with air shower scintillator arrays to separate gamma initiated showers from nuclei initiated showers, e.g. CASA-MIA [46], CYGNUS [47], KASCADE [48] or Ooty [49]. Only upper limits on gamma ray fluxes have been published.

The $\mathrm{L} 3+\mathrm{C}$ detector, Fig. 1, is located at a shallow depth of $30 \mathrm{~m}$. Therefore the muon energy-threshold is around $15 \mathrm{GeV}$. Larger values can be selected off-line. This opportunity has been taken in a previous work [50,51], in order to increase the significance of a possible signal. In this first attempt no signal was found in fixed time windows of one day, one or several months.

This article reports the observation through underground muons of a very high energy and intense flare emitted from a fixed direction in the northern hemisphere. It was recorded in August 2000 for a short time period of some three days. Contrary to the first search cited above, the sensitivity of this search is improved: The total number of reconstructed events analysed is increased, the event reconstruction is implemented in order to detect also muons crossing different chamber octants (see below), the data selectioncriteria are modified and a completely independent new analysis with a finer background determination is applied.

Below a short description of the $\mathrm{L} 3+\mathrm{C}$ detector is given, followed by the analysis of the sky survey data collected between July and November 1999, and April to November 2000. At the end, the results, together with a flux estimation and a discussion of the identification of a possible source, are given. 


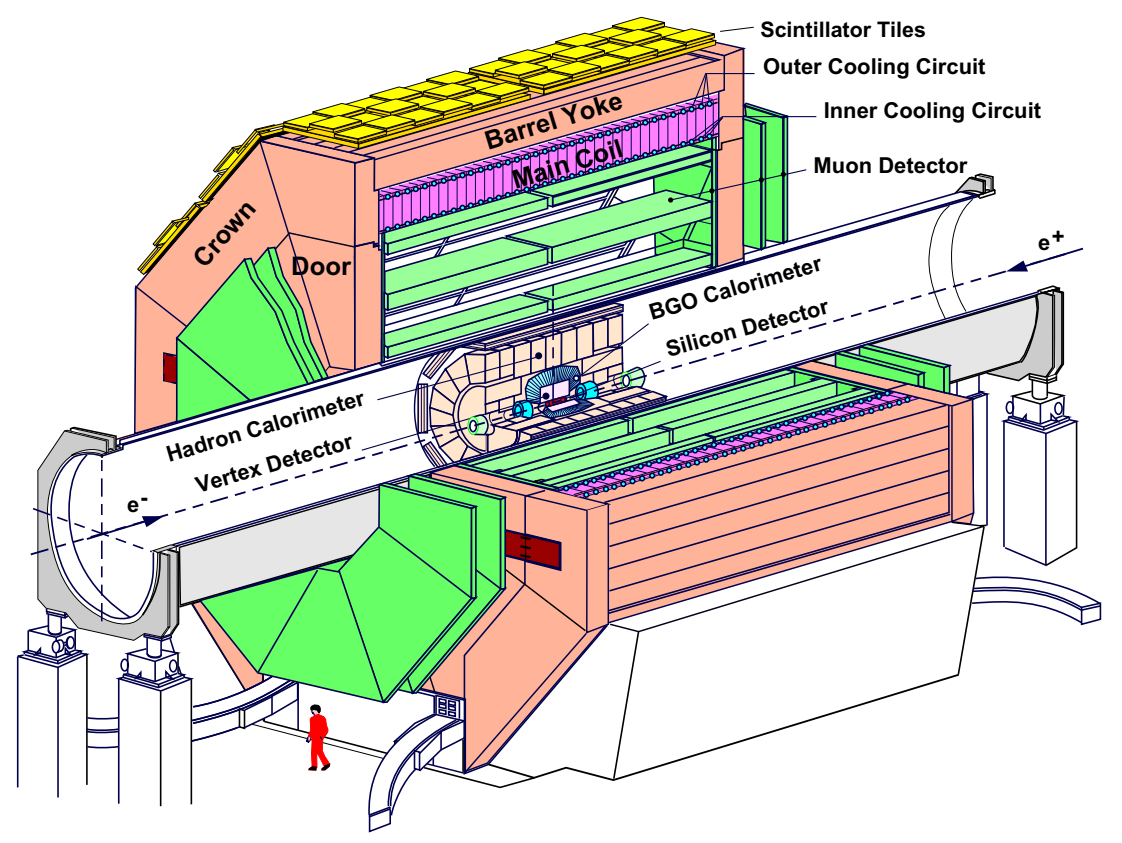

Fig. 1. The $\mathrm{L} 3+\mathrm{C}$ detector.

\section{The $\mathrm{L} 3+\mathrm{C}$ detector}

The $\mathrm{L} 3+\mathrm{C}$ spectrometer installed at the Large Electron-Positron collider, LEP at CERN, Geneva, has been described in [35]. Three layers of orthogonally arranged drift chambers are mounted inside a $0.5 \mathrm{~T}$ solenoidal magnet of $1000 \mathrm{~m}^{3}$ volume which allow the measurement of the momenta of muons precisely (Fig. 2) [52]. In the present analysis muons crossing at least any three chambers are selected. To record the arrival time of cosmic muons $202 \mathrm{~m}^{2}$ of scintillators are installed on the top of the iron yoke. The detector is located $450 \mathrm{~m}$ above sea level, at a latitude of $46.25^{\circ} \mathrm{N}$ and a longitude of $6.02^{\circ} \mathrm{E}$, and $30 \mathrm{~m}$ below ground. The energy threshold for vertically incident muons is around $15 \mathrm{GeV}$. The angular resolution and the pointing precision has been determined by the observation of the Moon shadow $[53,54]$. At muon energies above $65 \mathrm{GeV}$ the single muon angular resolution has been measured to be $0.28^{\circ}$ and the pointing precision to be better than $0.1^{\circ}$.

The acceptance of the $\mathrm{L} 3+\mathrm{C}$ detector for primary protons and gamma rays as a function of their energy and angular distribution has been calculated with a Monte Carlo simulation based on primary proton-, nuclei-, and gamma-energy spectra according to [55].

The simulation program can be divided into two parts: the muon generator, in which the CORSIKA code [56] (QGSJET01 interaction model [57]) is used to trace the cascade in the atmosphere; the detector simulator, built on the frame of GEANT3 [58] which tracks the muon in the molasse overburden and the detector, records the signals fired in the scintillator and the chambers. The reconstruction of the events is done in the same way as for real muons.

Three categories of primaries are simulated: (i) isotropically incident nuclei with fluxes and power law spectra adopted from [55], (ii) isotropically incident gamma rays with different spectral slopes, and (iii) gamma rays originating from a source with different spectral slopes.

The CORSIKA-QGSJET model cannot reproduce precisely the observed ground level muon fluxes [36] originating from primary nuclei. This defect has been amended by applying an efficiency

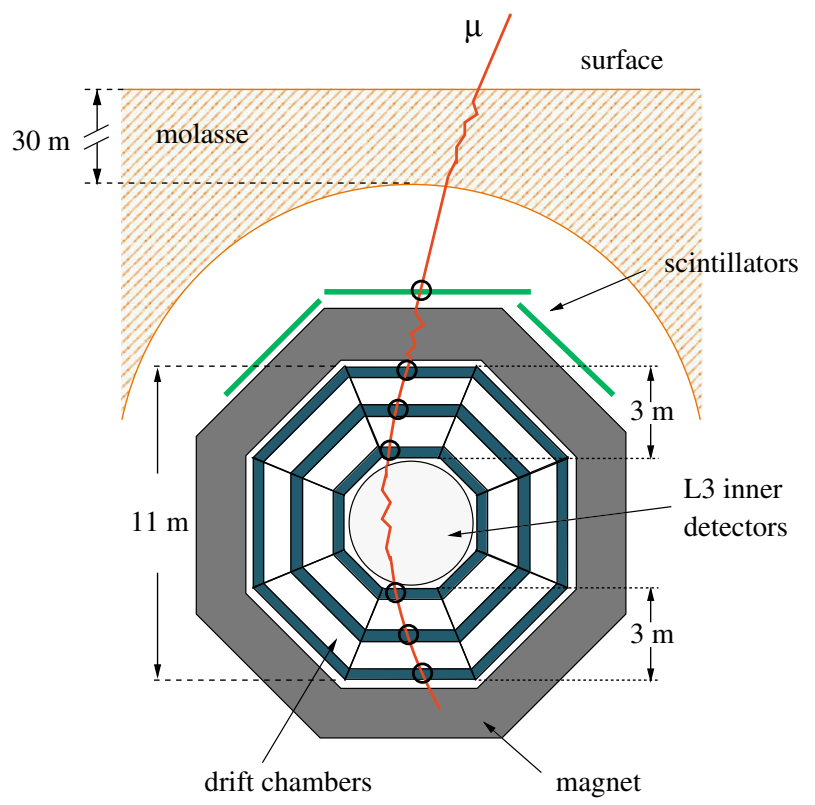

Fig. 2. Tracking of a muon through the $\mathrm{L} 3+\mathrm{C}$ detector.

curve to the simulated muon spectrum at the ground level. This amendment is not applied for primary gamma rays. The zenith distribution of generated muons agrees well with the measurement [36]

Fig. 3 shows the calculated vertical acceptance of the $\mathrm{L} 3+\mathrm{C}$ detector for primary protons and gammas as a function of their energy. The detector accepts events down to zenith angles of $58^{\circ}$. In the energy region $20-300 \mathrm{GeV}$, which is not yet fully explored by gamma ray astronomy experiments so far, $\mathrm{L} 3+\mathrm{C}$ has a certain acceptance. At the same time it still exhibits a good angular resolution.

The zenith- and azimuth-angle of the detected muon is used to determine the incoming direction of its primary on the top of the atmosphere. The angular resolutions $\sigma_{\text {res }}$ of gamma primaries 


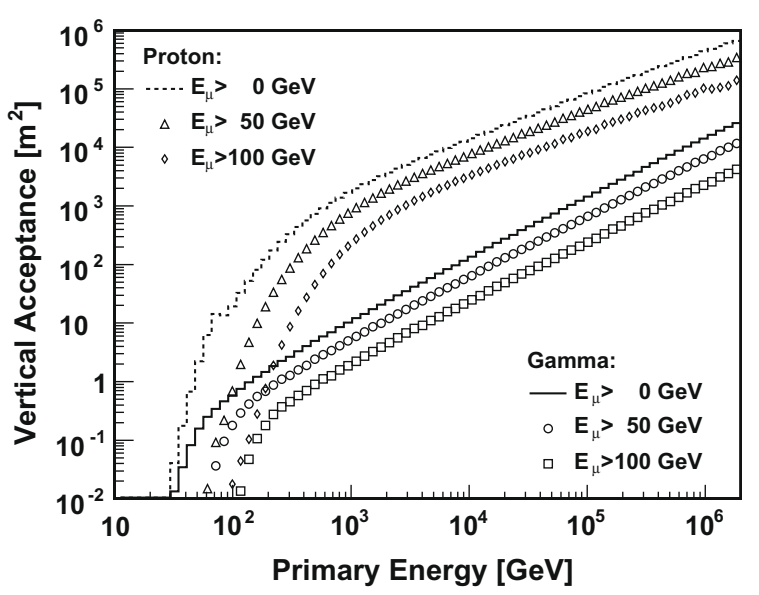

Fig. 3. The vertical acceptance (within a zenith angle of $15^{\circ}$ ) of the $\mathrm{L} 3+\mathrm{C}$ muon spectrometer for muons with threshold energies 0,50 , and $100 \mathrm{GeV}$ at ground level, as a function of the primary energy for protons and gammas, respectively. The acceptance in units $\left[\mathrm{m}^{2}\right]$ is defined as "effective area" = number of muons $\mathrm{N}_{\mu} /\left(I_{\gamma}\left(>E_{\gamma}\right) \times \Delta t\right)$ for gammas originating from a point source, and $=\mathrm{N}_{\mu} /\left(I_{p}\right.$ $\left.\left(>E_{p}\right) \times \Delta \boldsymbol{\Omega} \times \Delta t\right)$ for isotropically incident protons ( $\Delta \boldsymbol{\Omega}=$ solid angle, $I_{\gamma}\left(>E_{\gamma}\right), I_{p}\left(>E_{p}\right)=$ integral gamma flux, respectively integral proton flux). For curves labelled " $\mathrm{E}_{\mu}>0 \mathrm{GeV}$ " no energy cut has been applied.

at different zenith angles are given in Table 1 . It has been obtained by recording so-called "di-muon events". These are events with a simultaneous observation of two parallel tracks. High energy muons originating from far above the detector are supposed to be parallel. Thus the angular resolution can be measured and be compared to the MC simulation. Fig. 4 shows the comparison of the opening angle of the di-muon events of MC and data for different muon energy thresholds.

In order to get a most significant signal the bin sizes have been optimized with the help of a toy Monte Carlo program for different slopes of the assumed gamma spectra. These optimized bin sizes for square bins $W_{\text {opt }}$ are also listed in Table 1, under the assumption that the background content is large enough.

\section{Search procedure}

The method to search for short bursts of muon events pointing back to given sky regions is explained in detail in the following subsections. It starts with the selection of good quality muon tracks. Then different time binnings and sets of sky cell maps are defined. Event excesses for particular time and cell sizes are detected by determining the significance of a possible signal with respect to the background. The number of background events being space and time dependent, a special procedure has been developed to know precisely the expected number of background counts for a given time interval in each sky cell (see below).

\subsection{Event selection}

Two major selection criteria have been applied on the raw data set:

- Live-time selection: Data are collected in runs lasting typically $20 \mathrm{~min}$. The events are binned in time periods of $0.84 \mathrm{~s}$, for which a live-time counter assures the availability of the set-up to accept triggers. The basic input to the analysis is thus the number of events in bins of this length. If the number of events in any live-time bin of a given run deviates statistically by more than 4 standard deviations (sd) from the mean observed in the bins of runs before and after the run under test, the bin is excluded. A run with a percentage over $25 \%$ of excluded bins is discarded. The stability of the magnetic field is verified for each live-time bin by evaluating the ratio of the number of events with momenta larger than $50 \mathrm{GeV} / \mathrm{c}$ and the total number of events. A single deviation of more than 5.5 sd forces the cancellation of the particular run. Altogether around $1 \%$ of all runs were eliminated from the analysis.

- Track selection: In order to select only events with good momentum- and angular-resolution, at least one track crossing at least three muon chambers in the bending plane ("P-chambers"), two layers in the non-bending plane (" $Z$-chambers") of the magnetic field, and producing a scintillator signal is requested. To ensure a good track quality, relatively loose cuts are also applied to some parameters, such as the $\chi^{2}$ of the track fitting, and the variance of the muon direction obtained by a backtracking method. Moreover, the measured muon momentum is requested to be greater than $3 \mathrm{GeV} / \mathrm{c}$ at the detector level.

After the application of the mentioned selection criteria $2.9 \times 10^{9}$ events out of the total data set remain, and the effective live-time is 252.4 days evenly distributed over the 330 days of data taking.

\subsection{The time binning}

The purpose of this study is to find short bursts of muon events. Therefore the whole data-taking period is divided into equal time bins of given length. For the present analysis 12 time bins are chosen, lasting $2^{\mathrm{m}}$ minutes with $m$ ranging from 1 to 12 . That is to say, the data are repeatedly used to search for signals with 12 different time scales, or trials, scanning time windows between 2 minutes and 2.8 days. If a burst would be found, longer time bins would also be analyzed.

All time binnings start at 00:00:00:01:01:1999 UTC.

\subsection{Mapping the sky}

The sky is subdivided into rectangular cells in the following manner: The declination, $\delta$, is divided into $n$ small bands $\delta_{i}(i=1,2, \ldots, n)$ with equal width $\Delta \delta=180^{\circ} / n$. For each declination band $\delta_{i}$, the right ascension, $\alpha$, is further divided to get $m_{i}$ cells

Table 1

Angular resolutions $\left(\sigma_{\text {res }}\right)$ of gamma primaries and optimized bin sizes $W_{\text {opt }}$ at different zenith angles and for different slopes of the gamma spectrum.

\begin{tabular}{|c|c|c|c|c|c|c|c|c|c|c|c|c|}
\hline \multirow[t]{2}{*}{ Zenith } & \multicolumn{2}{|c|}{ Slope: -2.5} & \multicolumn{2}{|c|}{ Slope: -3.0} & \multicolumn{2}{|c|}{ Slope: -3.5} & \multicolumn{2}{|c|}{ Slope: -4.0} & \multicolumn{2}{|c|}{ Slope: -4.5} & \multicolumn{2}{|c|}{ Slope: -5.0} \\
\hline & $\sigma_{\text {res }}$ & $W_{\mathrm{opt}}$ & $\sigma_{\text {res }}$ & $W_{\mathrm{opt}}$ & $\sigma_{\text {res }}$ & $W_{\mathrm{opt}}$ & $\sigma_{\text {res }}$ & $W_{\mathrm{opt}}$ & $\sigma_{\text {res }}$ & $W_{\mathrm{opt}}$ & $\sigma_{\text {res }}$ & $W_{\text {opt }}$ \\
\hline $0^{\circ}-10^{\circ}$ & $0.49^{\circ}$ & $0.68^{\circ}$ & $0.67^{\circ}$ & $0.89^{\circ}$ & $0.85^{\circ}$ & $1.15^{\circ}$ & $0.97^{\circ}$ & $1.32^{\circ}$ & $1.09^{\circ}$ & $1.50^{\circ}$ & $1.21^{\circ}$ & $1.62^{\circ}$ \\
\hline $10^{\circ}-20^{\circ}$ & $0.49^{\circ}$ & $0.67^{\circ}$ & $0.65^{\circ}$ & $0.88^{\circ}$ & $0.81^{\circ}$ & $1.09^{\circ}$ & $0.95^{\circ}$ & $1.26^{\circ}$ & $1.06^{\circ}$ & $1.42^{\circ}$ & $1.17^{\circ}$ & $1.59^{\circ}$ \\
\hline $20^{\circ}-30^{\circ}$ & $0.48^{\circ}$ & $0.66^{\circ}$ & $0.62^{\circ}$ & $0.86^{\circ}$ & $0.80^{\circ}$ & $1.06^{\circ}$ & $0.94^{\circ}$ & $1.26^{\circ}$ & $1.06^{\circ}$ & $1.44^{\circ}$ & $1.18^{\circ}$ & $1.60^{\circ}$ \\
\hline $30^{\circ}-40^{\circ}$ & $0.46^{\circ}$ & $0.63^{\circ}$ & $0.61^{\circ}$ & $0.83^{\circ}$ & $0.78^{\circ}$ & $1.04^{\circ}$ & $0.91^{\circ}$ & $1.23^{\circ}$ & $1.04^{\circ}$ & $1.39^{\circ}$ & $1.14^{\circ}$ & $1.53^{\circ}$ \\
\hline $40^{\circ}-50^{\circ}$ & $0.42^{\circ}$ & $0.58^{\circ}$ & $0.56^{\circ}$ & $0.76^{\circ}$ & $0.70^{\circ}$ & $0.93^{\circ}$ & $0.83^{\circ}$ & $1.10^{\circ}$ & $0.95^{\circ}$ & $1.25^{\circ}$ & $1.05^{\circ}$ & $1.42^{\circ}$ \\
\hline $50^{\circ}-60^{\circ}$ & $0.38^{\circ}$ & $0.53^{\circ}$ & $0.48^{\circ}$ & $0.67^{\circ}$ & $0.60^{\circ}$ & $0.81^{\circ}$ & $0.71^{\circ}$ & $0.93^{\circ}$ & $0.78^{\circ}$ & $1.06^{\circ}$ & $0.89^{\circ}$ & $1.15^{\circ}$ \\
\hline
\end{tabular}



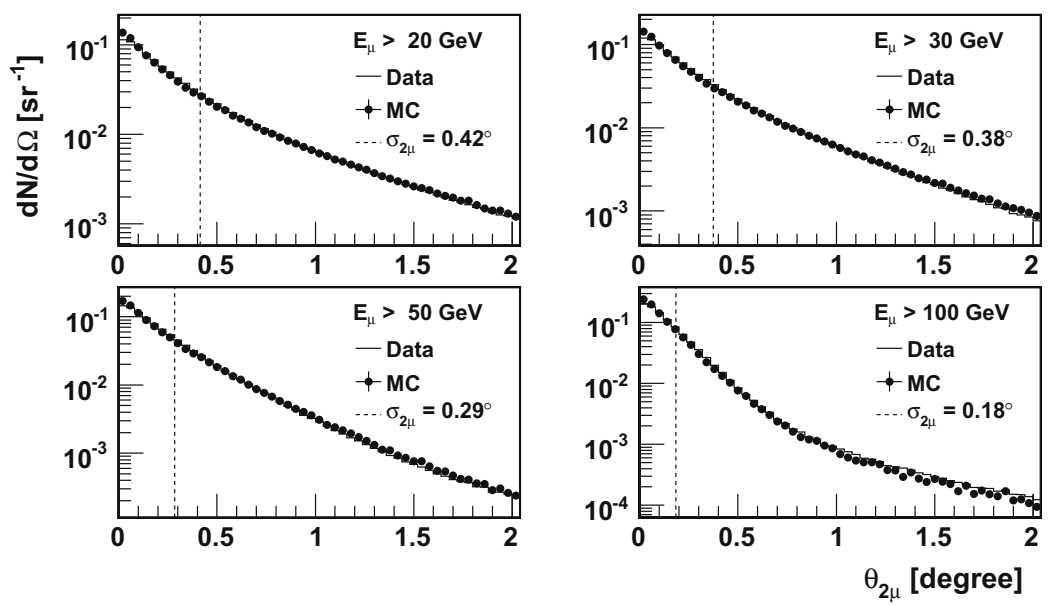

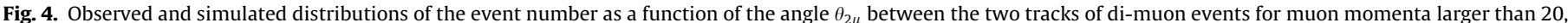
30, 50 and $100 \mathrm{GeV}$. The assumed primary composition and the spectral index of the flux are taken from [55].

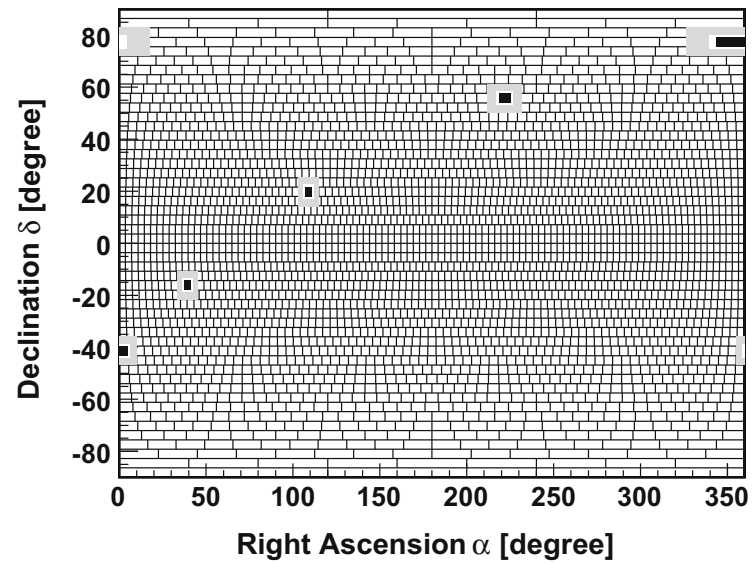

Fig. 5. An example of a grid of sky cells. Shown are also some selected cells surrounded by their "background region" (hollow rectangles).

with width $\Delta \alpha_{i}$, where $m_{i}$ is the best integer to satisfy $360^{\circ} / m_{i}=\Delta \delta / \cos \delta_{i}$, and $\Delta \alpha_{i}=360^{\circ} / m_{i}$. Fig. 5 shows one example of a chosen grid with all its sky cells.

$\Delta \delta$ (or $n$ ) is the only parameter used for the definition of a sky cell. It should be set in order to match the optimized bin size calculated from the angular resolutions. However, there are many reasons to iterate the values $\Delta \delta$ : (i) the angular resolution depends not only on the energy spectrum of the signal, but also on the zenith angle; (ii) for small numbers of background-events, the optimized bin size depends also on the intensity of the signal; (iii) the source may not exactly reside in the center of the cell. Six $\Delta \delta$ values are therefore used: $1^{\circ}, 1.5^{\circ}, 2^{\circ}, 2.5^{\circ}, 3^{\circ}, 3.6^{\circ}$. But, in order to avoid too many trials, an a posteriori optimisation has been applied (see Section 3.6).

\subsection{Determination of the background in each cell}

Because of the varying LEP radiation and detector problems, the efficiency of the $\mathrm{L} 3+\mathrm{C}$ detector is not absolutely constant. Randomly occurring overall, as well as directionally dependent changes are observed. Methods to determine the background, such as the "time shuffling" [59], or "direct integral" [60,61] are therefore not applicable for the short flare search (the acceptance $A=A(h, \delta, t)$ cannot be factorized as $A=A(h, \delta) \times T(t)$, where $h$ equals the hour angle, $\delta$ equals the declination angle, and $t$ is the time).
The principle of the method used here relies on the fact that the ratio $1 / R$ of events from a region located around the cell of interest to the number of events in this particular cell, remains constant for a fixed direction with respect to the detector (except for the event of a flare signal), and can be measured accurately over the whole period of the experiment. By measuring the amount of events in the surrounding region within the same time interval, and knowing $R$, allows for the calculation of the expected background event number at the given time in the central cell of interest.

Explained in more details: The number of events in a region surrounding each sky cell at a short distance (in order to avoid the possibility that a signal - which may be present - influences the background) - called "background region" - is used to determine the number of background events in the particular sky cell under discussion after the following procedure has been established: the background region is defined as a hollow rectangle around the sky cell of interest; the half width ranges from $w_{a}=0.75 \times$ $\Delta \delta$ to $w_{b}=\Delta \delta+1^{\circ}$ in declination, and from $w_{a} \times\left(\Delta \alpha_{i} / \Delta \delta\right)$ to $w_{b} \times\left(\Delta \alpha_{i} / \Delta \delta\right)$ in right ascension. Some examples of background regions are shown shaded in Fig. 5. The solid angle ratio $R_{\text {geom }}$ of the sky cell and its background region can be precisely calculated, and amounts to $1 / 33.8,1 / 19.5,1 / 13.8,1 / 10.7,1 / 8.9$ and $1 / 7.4$, respectively for the six cell sizes. The number of events found in the background region cannot be directly converted into the number of background events corresponding to the center cell of interest. When the ensemble of windows - the sky cell and its background region - move on the celestial sphere in the local equatorial coordinate system (LECS), the acceptance ratio between the two windows is changing. But, when the two windows reside in a fixed position with respect to the LECS, the acceptance ratio should hardly depend on the efficiency changes of the detector - unless the efficiency turns to zero. The large statistics of the whole year's data is therefore used to calibrate the acceptance ratio, and a database containing all the acceptance ratios $(R(h, \delta))$ for every direction of LECS is established. Taking into account the fact that there are six different sizes of sky cells, six corresponding databases are created.

Each specific time bin under investigation is divided into several two minutes steps for which the acceptance ratio is available from the database.

To obtain the number of background events of the central cell the number of observed background events in the "background" cells is multiplied with this acceptance ratio. Summing up the number of background events for all the steps, the number of background events for the whole time bin is found. 
The systematic error of the acceptance ratio can be neglected, except for sky cells around the equatorial North pole, which are excluded from the search. The uncertainty of the significance caused by the statistical error of the acceptance ratio is estimated to be less than 0.2 standard deviation (sd).

In order to avoid the influence of the signal to the acceptance ratio, the data around the time bin under investigation from five time bins before, to five time bins after, are not used for the calibration of the acceptance ratio. So in fact, the databases are dynamical databases.

\subsection{Significance}

For each sky cell and time bin, the widely used Li-Ma prescription [62] (method of hypotheses test, based on [63-65]) is used to calculate the significance (with chance probability $P_{\text {sig. }}$ ) of the signal. Because the number of background events is determined from a much bigger background region, the statistical fluctuations are small. The $\alpha$ - "parameter" in the Li-Ma formula (1) below (defined there as the ratio of the on-source time to the off-source time) is therefore replaced by the above-cited solid angle ratios $R_{\text {geom }}, N_{\text {off }} \times \alpha$ is set to the number of background events in the cell under consideration, and $N_{\text {on }}$ is set to the total number of events in this same cell (compared to the cited procedure we transform the "time" related background determination into a "space" related background estimation).

\section{Significance}

$$
=\sqrt{2} \cdot\left\{N_{\text {on }} \cdot \ln \left[\frac{1+\alpha}{\alpha} \cdot \frac{N_{\text {on }}}{N_{\text {on }}+N_{\text {off }}}\right]+N_{\text {off }} \cdot \ln \left[(1+\alpha) \frac{N_{\text {off }}}{N_{\text {on }}+N_{\text {off }}}\right]\right\}^{1 / 2}
$$

If the number of background events in the particular cell of interest determined from the background region is zero, the formula is mathematically invalid. In this case the time bin is marked to be ineffective. This happens when the center of the sky cell is below the horizon during the considered time bin (in fact no cut on the zenith angle is applied to the data), or the live-time is zero, or in rare cases when the number of events in the background region is vanishing. This factor of ineffectiveness is large for short time binnings. The sky cell too is marked to be ineffective if for a given time binning and a given cell size the number of effective time bins is zero.

The Li-Ma method is not appropriate for the detection of very small signals. However, in this study, for small time binnings (that is to say, low number of background events), the number of trials becomes big. Thus, if an excess of events is to be justified, the number of excess events must be large enough. The method is then applicable.

\subsection{Selection of candidates}

The number of time bins analyzed for the 12 time binnings amounts to $4.8 \times 10^{5}$, and the number of sky cells for the 6 choices of sky cell sizes is $8.5 \times 10^{4}$, giving a total of $4.0 \times 10^{10}$ observations. About $82 \%$ of all cells with chosen time windows are ineffective (see Section 3.4) (observing 58\% of the sky 32\% of the time).

Accounting for all trials for the remaining "effective" $18 \%$ of the cases, a signal with a chance probability to be a statistical fluctuation of less than $1 / 2 \times 2.7 \times 10^{-3}$ ( 3 sd level), requires a $7.2 \mathrm{sd}$ significance for an excess in any given sky cell. In reality one is faced with physical conditions which do not allow for a uniform treatment of all sky cells and time binnings. The "standard" way just described cannot be applied for the present search and set of data. The following procedure is therefore proposed: [In the following a particular numerical example is given in square brackets for all discussed quantities.]

\subsubsection{Selecting the time bin}

For given time binning $\left[2^{4} \mathrm{~min}\right]$ and for given cell size $\left[1^{\circ}\right]$ the number of time bins for each sky cell is determined individually. Only the time bin with the most significant excess is selected out of the total number of time bins $N_{\text {time_bin }}\left[29,700=\frac{330 d}{2^{4} \mathrm{~min}}\right]$. A sky map containing all the cells with the largest significance is established, including the number of effective time bins $N_{\text {time_bin }}^{\text {eff }}\left[11537=29700 \times\left(2 \times 70^{\circ} / 360^{\circ}\right)\right.$, where $70^{\circ}$ is the maximal zenith angle used for the analysis. - Remark: for short time binning $\left.N_{\text {time_bin }}^{\text {eff }} \ll N_{\text {time_bin }}\right]$. In total $12 \times 6=72$ maps $M_{j},(j=$ $1,2, \ldots, 72)$ are produced, corresponding to the twelve choices of time windows and the six sizes of cells.

For each sky cell $c_{i}$ of the map $M_{j}$ containing a total of $N_{\text {sky_cell }}\left(M_{j}\right)\left[10,314=4 \times \pi /\left(2^{\circ} \times 2^{\circ}\right)\right]$ sky cells the chance occurrence $Q\left(c_{i}, M_{j}\right)$ (defined here as the expected event number) of a fluctuation of the background is calculated according to

$Q\left(c_{i}, M_{j}\right)=Q_{\text {sig }}\left(c_{i}\right) \times N_{\text {time_bin }}^{\text {eff }}\left(c_{i}\right)$,

$\left[1.73 \times 10^{-6}=1.5 \times 10^{-10} \times 11,537\right.$

where $Q_{\text {sig }}\left(c_{i}\right)=-\ln \cdot\left(1-P_{\text {sig }}\right)$, is the chance occurrence corresponding to the particular significance of the most significant time bin. It is a concept particularly introduced in this analysis to take the number of trials into account. According to the nature of the Poisson distribution, the chance occurrence $Q$ is related to the chance probability $P$ as just given, or as $P=1-e^{-Q}$.

For a given map the total number of effective cells $N_{\text {sky_cell }}^{\text {eff }}\left(M_{j}\right)[6049 \simeq 10314 \times 58.6 \%$, where $58.6 \%$ is the fraction of the $\mathrm{L} 3+\mathrm{C}$ field of view out of $4 \pi$ ] is obtained by recording only the cells containing at least one effective time bin.

The chance occurence for each sky cell among all effective cells is finally calculated:

$Q^{\text {sum }}\left(c_{i}, M_{j}\right)=Q\left(c_{i}, M_{j}\right) \times N_{\text {sky_cell }}^{\text {eff }}\left(M_{j}\right)$

$\left[1.05 \times 10^{-2}=1.73 \times 10^{-6} \times 6049\right]$

The 72 maps are equally treated; no physics model indicates that some time binning, or some sky cell size, should be favoured.

\subsubsection{Selecting the cell size}

As mentioned, for low background event numbers, the cell size optimisation depends also on the intensity of the signal (besides of the angular resolution of the detector). "Toy" Monte Carlo (MC) simulations to get the optimized bin size for each cell needs to be done for several models of an assumed primary gamma spectrum (see Chapter 2). In order to get the statistics of some real observations out of all effective cells of a given map, only $n=5$ sky cells with the smallest chance occurrence are retained in each sky map.

The result shows that, on one hand, the dependence of the optimised bin size to the statistics cannot be neglected, but on the other hand, the significance is not very sensitive to the bin size when it is not too far from the optimized value. With respect also of the large uncertainty on the optimized bin size caused by the

Table 2

The selected cell sizes for the different time binnings.

\begin{tabular}{ll}
\hline Time binning $(\mathrm{min})$ & Selected cell sizes \\
\hline $2^{1}-2^{4}$ & $2.0^{\circ}-3.0^{\circ}$ \\
$2^{5}-2^{8}$ & $1.5^{\circ}-2.5^{\circ}$ \\
$2^{9}-2^{12}$ & $1.0^{\circ}-2.0^{\circ}$ \\
\hline
\end{tabular}


Table 3

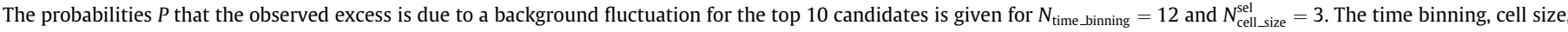
significance, corresponding chance probability, number of effective time bins, and of sky cells are given.

\begin{tabular}{|c|c|c|c|c|c|c|}
\hline Time binning (min) & Cell size & Significance & $P_{\text {sig }}$ & $N_{\text {time_bin }}^{\text {eff }}$ & $N_{\text {sky_cell }}^{\text {eff }}$ & $P$ \\
\hline $2^{12}$ & $2.0^{\circ}$ & 6.356 & $1.0 \times 10^{-10}$ & 115 & 6049 & $2.6 \times 10^{-3}$ \\
\hline $2^{4}$ & $2.0^{\circ}$ & 6.294 & $1.5 \times 10^{-10}$ & 11,537 & 6049 & $3.1 \times 10^{-1}$ \\
\hline $2^{5}$ & $1.5^{\circ}$ & 6.148 & $3.9 \times 10^{-10}$ & 6220 & 10596 & $6.0 \times 10^{-1}$ \\
\hline $2^{1}$ & $2.5^{\circ}$ & 6.125 & $4.5 \times 10^{-10}$ & 39,491 & 3868 & $9.2 \times 10^{-1}$ \\
\hline $2^{11}$ & $2.0^{\circ}$ & 5.297 & $5.9 \times 10^{-8}$ & 220 & 6049 & $9.4 \times 10^{-1}$ \\
\hline $2^{5}$ & $2.5^{\circ}$ & 5.757 & $4.3 \times 10^{-9}$ & 5724 & 3868 & $9.7 \times 10^{-1}$ \\
\hline $2^{2}$ & $2.0^{\circ}$ & 5.863 & $2.3 \times 10^{-9}$ & 12,752 & 6049 & 1.0 \\
\hline $2^{4}$ & $2.0^{\circ}$ & 5.698 & $6.1 \times 10^{-9}$ & 5297 & 6049 & 1.0 \\
\hline $2^{9}$ & $2.0^{\circ}$ & 5.311 & $5.4 \times 10^{-8}$ & 662 & 6049 & 1.0 \\
\hline $2^{8}$ & $2.0^{\circ}$ & 5.368 & $4.0 \times 10^{-8}$ & 1087 & 6049 & 1.0 \\
\hline
\end{tabular}

uncertain models of the primary spectrum, a general rule has been adopted on the selection of the cell sizes for all the signal candidates and is displayed in Table 2. Candidates with cell sizes out of the ranges set by the table are rejected.

For each time binning, there are therefore finally only $N_{\text {cell_size }}^{\text {sel }}=$ 3 cell sizes $(j=1,2, \ldots, 36)$.

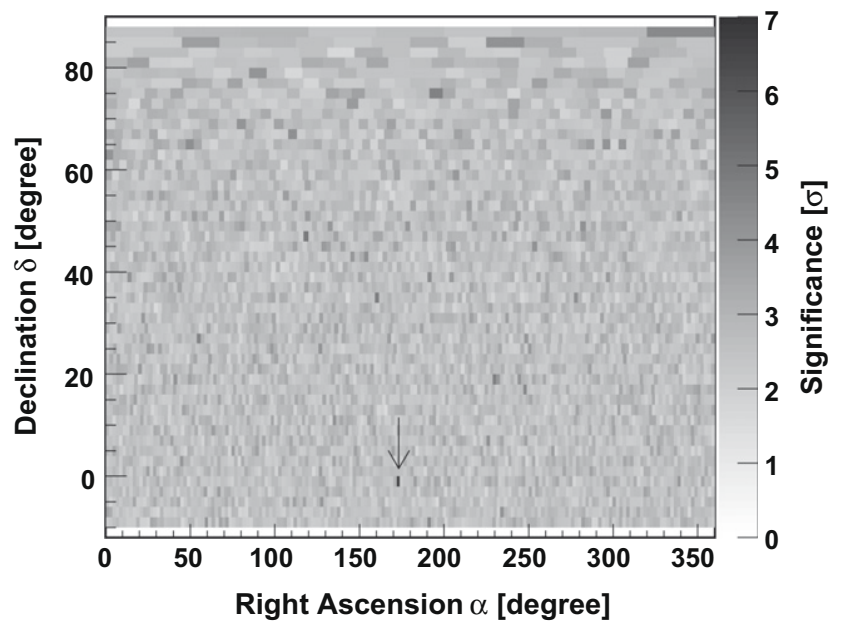

Fig. 6. The only sky map found exhibiting a cell with an excess of events with a chance probability smaller than $1 \%$ to be a background fluctuation (cell position: $\alpha=173^{\circ}, \delta=-1^{\circ}$.

\subsubsection{Selecting the most significant excess}

Based on the discussed procedure, the chance occurrence $Q^{\text {final }}\left(c_{i}, M_{j}\right)$ for each excess number of events in a given cell $c_{i}$ of map $M_{j}$ to be a statistical fluctuation of the background is calculated as

$$
\begin{aligned}
& Q^{\text {final }}\left(c_{i}, M_{j}\right)=Q^{\text {sum }}\left(c_{i}, M_{j}\right) \times N_{\text {time_binning }} \times N_{\text {cell_size }}^{\text {sel }}, \\
& \quad(j=1,2, \ldots, 36)\left[0.377=1.05 \times 10^{-2} \times 12 \times 3\right] .
\end{aligned}
$$

The chance occurrence $Q^{\text {final }}\left(c_{i}, M_{j}\right)$ is translated to a final chance probability $P[0.314=1-\exp (-0.377)]$.

Table 3 shows the actual values of the involved quantities for the calculation of the chance probabilities.

\section{Results}

One and only one candidate has been found from the sky survey described above having a chance probability of less than $1 \%$ to be a background fluctuation. Some characteristics of this observation are given below:

- Time binning $=2^{12} \min$.

- Sky cell size $=2.0^{\circ} \times 2.0^{\circ}$.

- Number of events inside cell $=726$, number of background events $=562.3 \pm 6.4$, excess $=163.7 \pm 6.4$.

- Significance $=6.356 \sigma(\mathrm{Li}-\mathrm{Ma}$ prescription $)$ for this particular sky map and time window size.
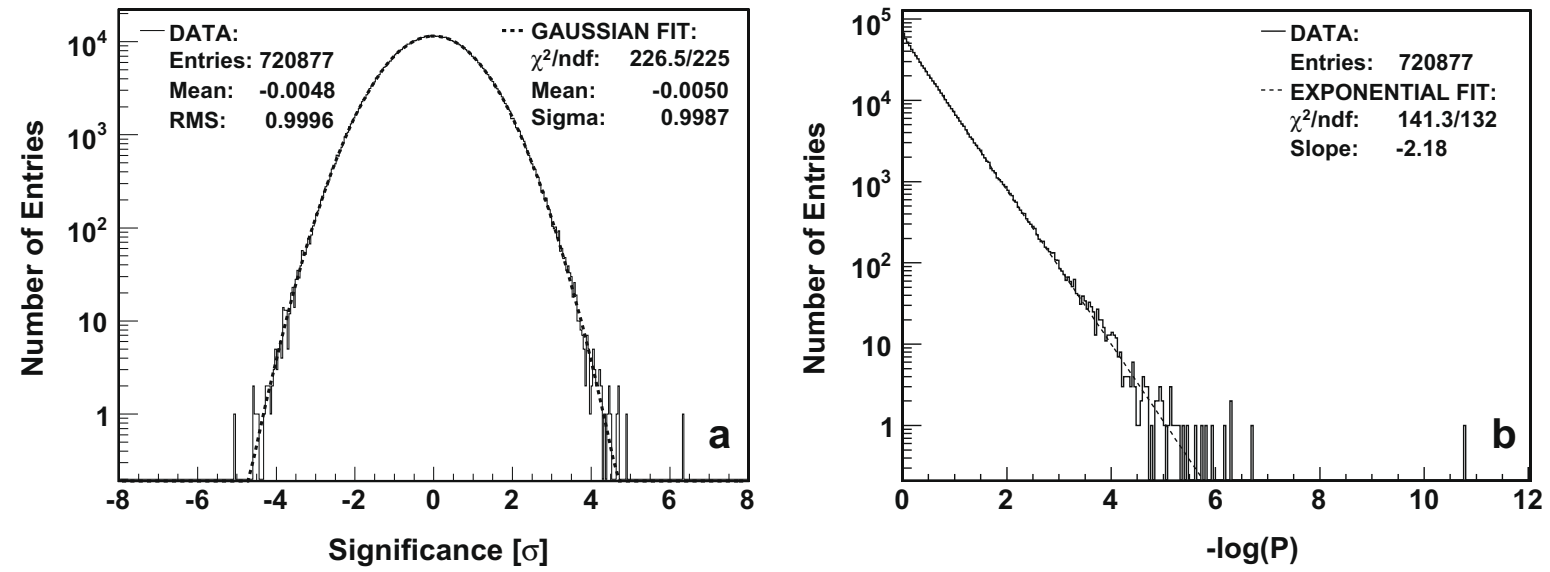

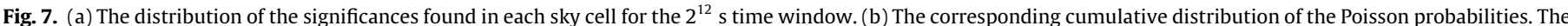

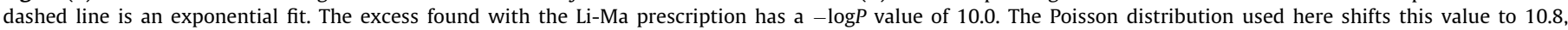
illustrating the non-appropriate distribution used here for simplicity. 


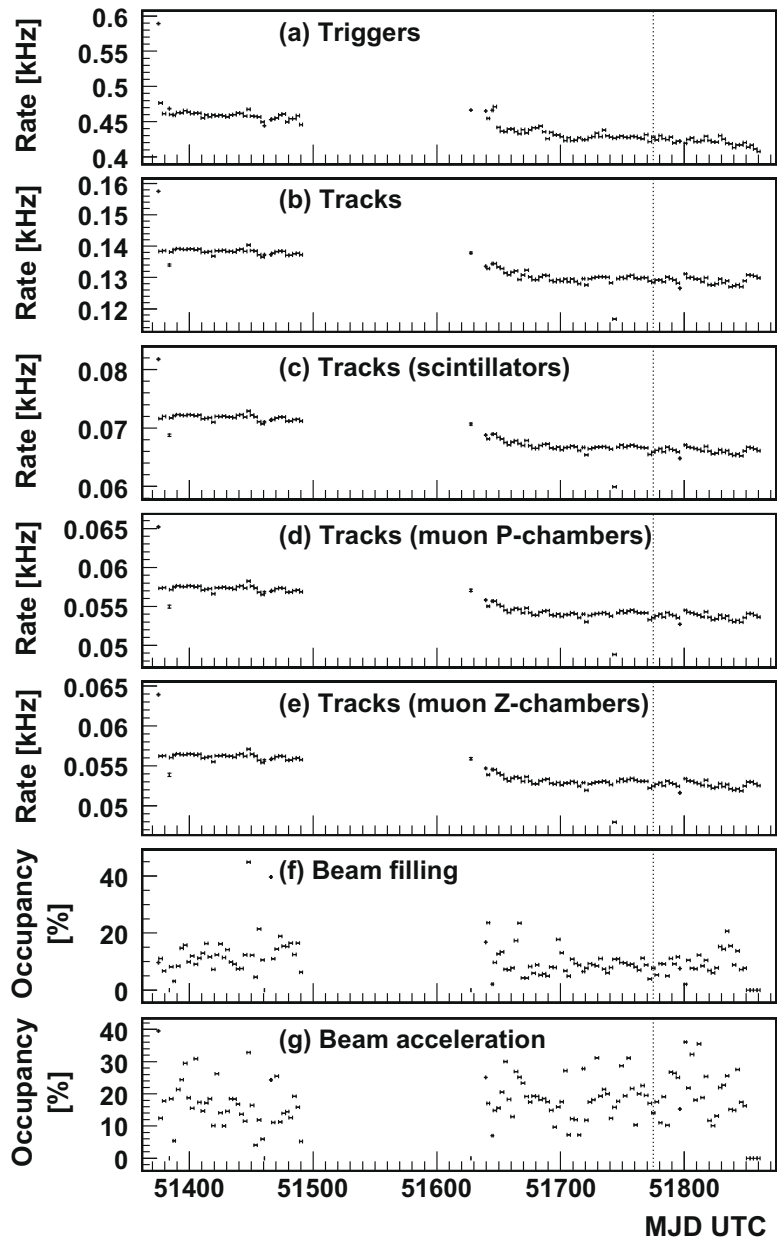

Fig. 8. For a time window set to $2^{12}$ min different quantities are displayed as a function of time. See the text for explanations. The vertical dotted line indicates the time of the recorded excess. The big gap between MJD $=51,490$ and 51,650 corresponds to the lack of data during this period of time (LEP off, L3 magnet off).

- Chance probability $P$ to observe a background fluctuation as an excess $=2.6 \times 10^{-3}$, when taking into account all trials, the different analyzed sky maps and time window sizes.

- Position: Center of sky cell: right ascension $\alpha=173^{\circ}$, declination $\delta=-1^{\circ}$.

- Time and date of excess: 51773.489-51776.333 MJD (Modified Julian Date), corresponding to the calendar date and UT time:

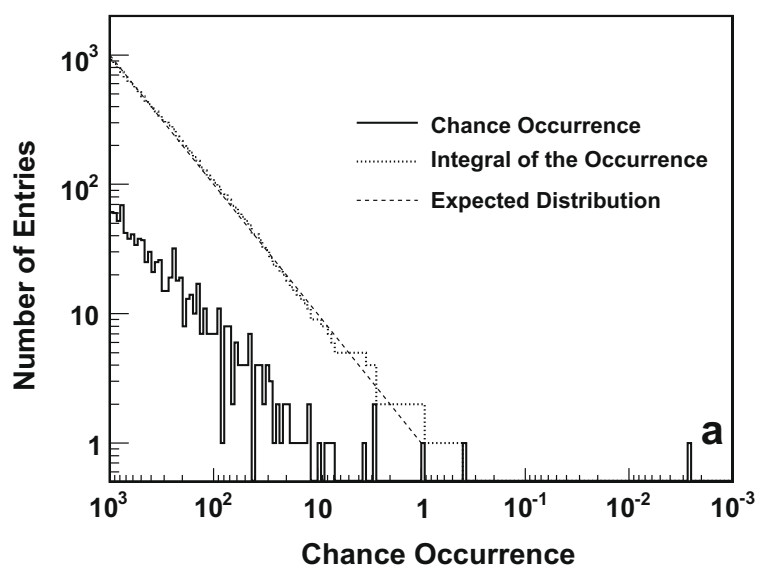

$11: 4417 / 08 / 2000$ to $08: 0020 / 08 / 2000$ (from the time evolution study the signal is observed to last up to 10 days; see below).

- Because of the low statistics no firm conclusion for the charge of the primary particle can be made from the observed $\boldsymbol{\mu}^{+} / \boldsymbol{\mu}^{-}$ charge ratio; but see Section 7.3.

The following figures illustrate the characteristics of the observed excess of events:

- Fig. 6 shows the significances found within each cell of the only sky map containing the excess presented above. The map contains all sky cells of size $2^{\circ} \times 2^{\circ}$ for a time window of 2.8 days $\left(=2^{12} \mathrm{~min}\right)$. The cell located at $\alpha=173^{\circ}$ and $\delta=-1^{\circ}$ is clearly identified as having the most important significance.

- Fig. 7a shows the distribution of all significances for the particular case of a time window of $2^{12}$ min and a cell size of $2.0^{\circ} \times 2.0^{\circ}$.

- Fig. 7b displays the cumulative Poisson probability to find a significant signal for the same conditions.

\section{Discussion of the experimental conditions}

The probability of $2.6 \times 10^{-3}$ for a chance occurrence of the observed excess being not negligible requires additional care. Problems with the detector behaviour, or the analysis, should be excluded and exceptional features of the signal found with respect to average background fluctuations registered.

Many different counting rate studies confirm the steady and good conditions of the detector and the data acquisition system during the particular time the event excess has been registered. Some examples are given below: Fig. 8a shows the stability observed of the trigger rate as a function of time. The vertical line indicates the time of the recorded excess. Similar distributions are shown for (b) the rate of reconstructed tracks, (c) the rate of tracks crossing the scintillators, $(\mathrm{d})$ the $P$ - and (e) the $Z$-chamber response of one chamber octant $(P$-chambers, respectively $Z$-chambers measure the momentum component perpendicular, respectively parallel to the magnetic field), (f) the occupancy of the data acquisition during LEP filling, and (g) the occupancy during the acceleration of the LEP beams. The accelerator running conditions during particular working modes may produce background signals, which may interfere with the data acquisition of the $\mathrm{L} 3+\mathrm{C}$ experiment. Therefore separate checks are made, that LEP radiation induced noise signals are not modifying the detector response, in particular during beam filling and acceleration. No particular problem is mentioned in the logbook or the database during the time of interest. More trivial errors, like counting events twice, have not been found (no events have the same GPS timing or the same combined zenith, azimuth and momentum).

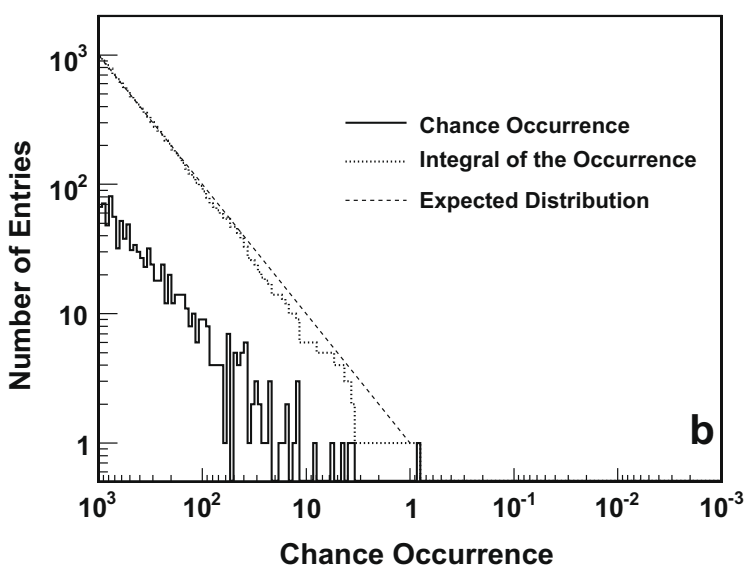

Fig. 9. The distribution of the chance occurrence for the top 1000 most significant excesses (a), and the top 1000 most significant deficits (b), respectively. 


\section{Discussion of the observation}

\subsection{Significance and chance probability}

The distributions of the significances (e.g. Fig. 7a) for any time binning and selected cell size show perfect Gaussian distributions (center at 0 , width $=1 \mathrm{sd}$ ). In cases of low statistics slight devia- tions on the negative side are due to the chosen Li-Ma prescription, and are unphysical.

The distribution of the chance occurrency for the excess is compared to the expected distribution ( $n$ is set equal to 100 in step (3.6.2) of Section 3.6, in order to select more candidates and getting better statistics for the expected distribution). The integral number of entries shows a perfect agreement with the expected cumulative
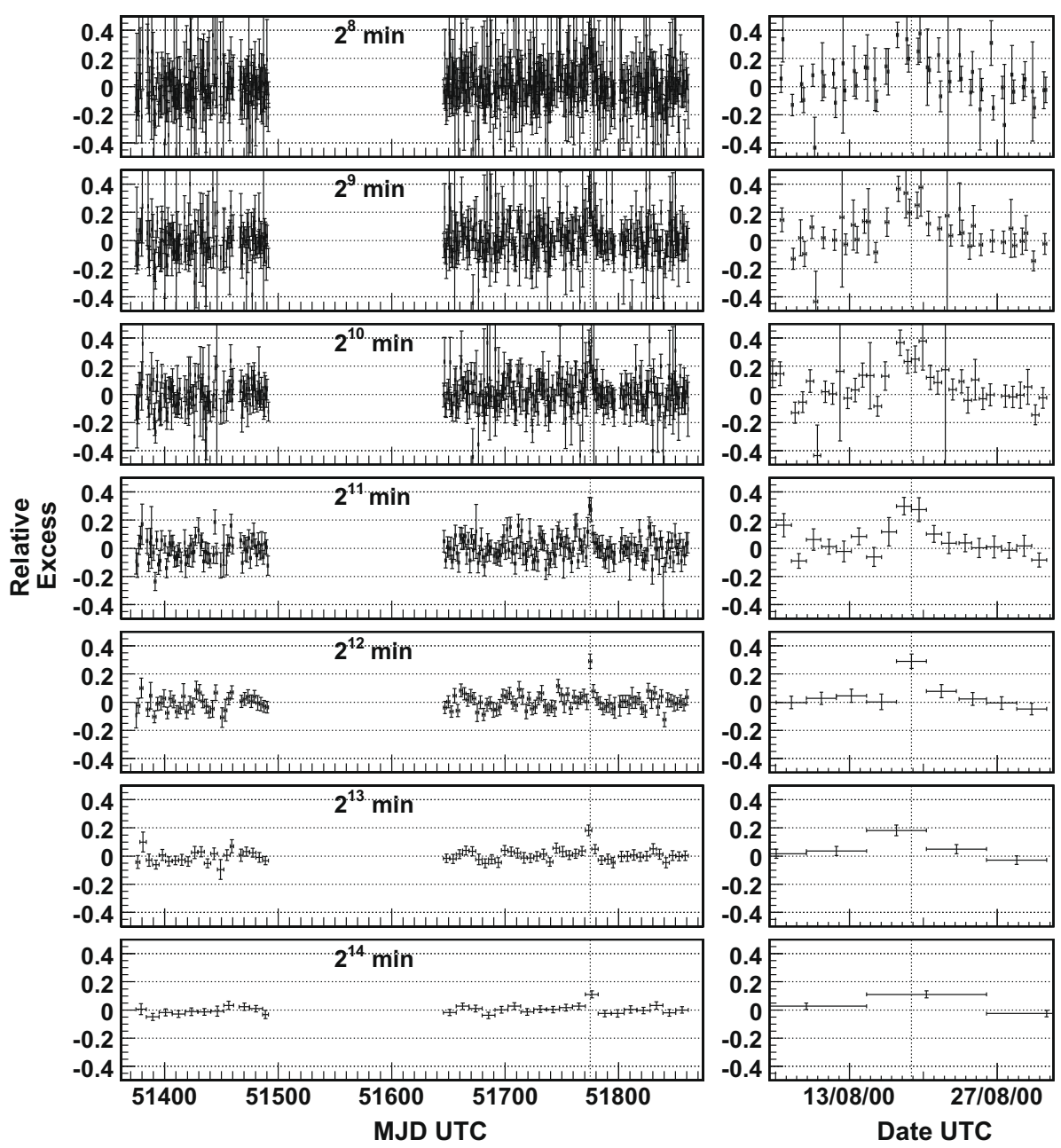

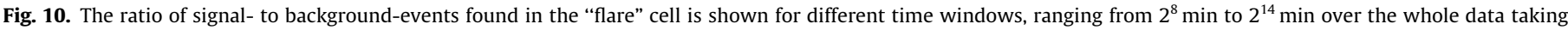

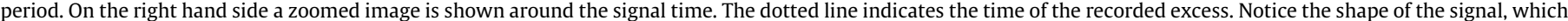
is asymmetric and corresponds to an expected sudden flare signal with some decay constant.

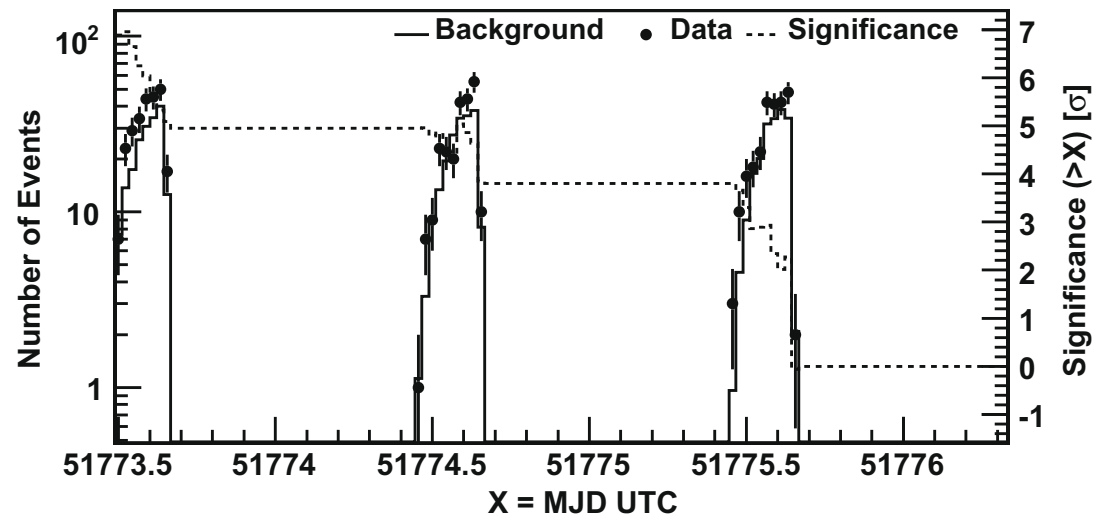

Fig. 11. Data and background as a function of the Modified Julian Date (MDJ). The integral significance is also plotted. 
distribution (Fig. 9a). The observed chance occurence of the excess is $2.6 \times 10^{-3}$, whereas the next most significant excess is equal to 0.39 .

Following the same procedure as above to find an excess, the distribution of the chance occurence for observing a deficit is also studied. Good "linearity" has been found too (Fig. 9b). The chance occurence of the most significant deficit is 0.82 .

\subsection{The evolution in time of the observed signal}

The sky cell containing the excess of events has been analyzed for each time binning. Fig. 10 displays the ratio of the number of excess events to the number of background events. It represents the "light-curve" of the candidate flare event. The asymmetric burst-like excess is an unlikely feature for a statistical fluctuation. The overall duration of the flare lasts for up to $\approx 3$ days, exhibiting a very rapid increase followed by an exponential decay.

Another way to look at the time evolution of the signal is given in Fig. 11. The number of data and background events are plotted as a function of the Modified Julian Date (MJD), together with the significance of the signal.

\subsection{The energy dependence of the signal}

A second indication that the excess is not due to a background fluctuation is obtained by varying the muon energy threshold, since one does not expect background event fluctuations observed at different energies to happen at the same time. Fig. 12 shows, that the signal persists at least up to a muon energy of $50 \mathrm{GeV}$, corresponding to a primary gamma energy of approximately $500 \mathrm{GeV}$.

Fig. 13 shows the event numbers (data and measured background) together with the integral significance, as a function of the ground level muon momenta recorded in the signal cell.

\subsection{The dependence on the chosen cell size}

As shown in Fig. 14 the signal persists when reducing the cellsize. This is an additional confirmation that the signal is not just a background fluctuation. However the significance becomes smaller and the most significant cell is a little bit off from the center of the $2.0^{\circ}$ cell. The significance seems to disappear in the case of the $1.5^{\circ}$, the $2.5^{\circ}$ and the $3.6^{\circ}$ cells. This is explained by the fact that in these
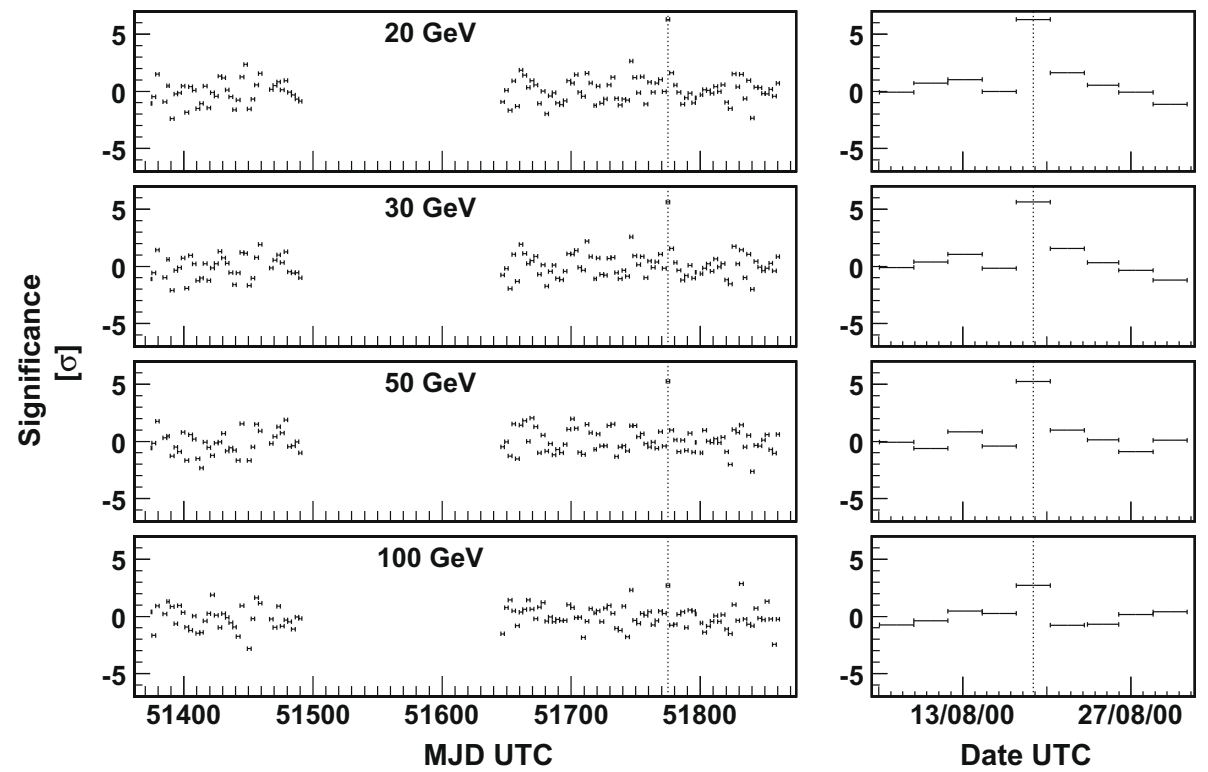

Fig. 12. The significance of the excess as a function of time is plotted for four different muon energy thresholds (time binning $=2^{12}$ min).

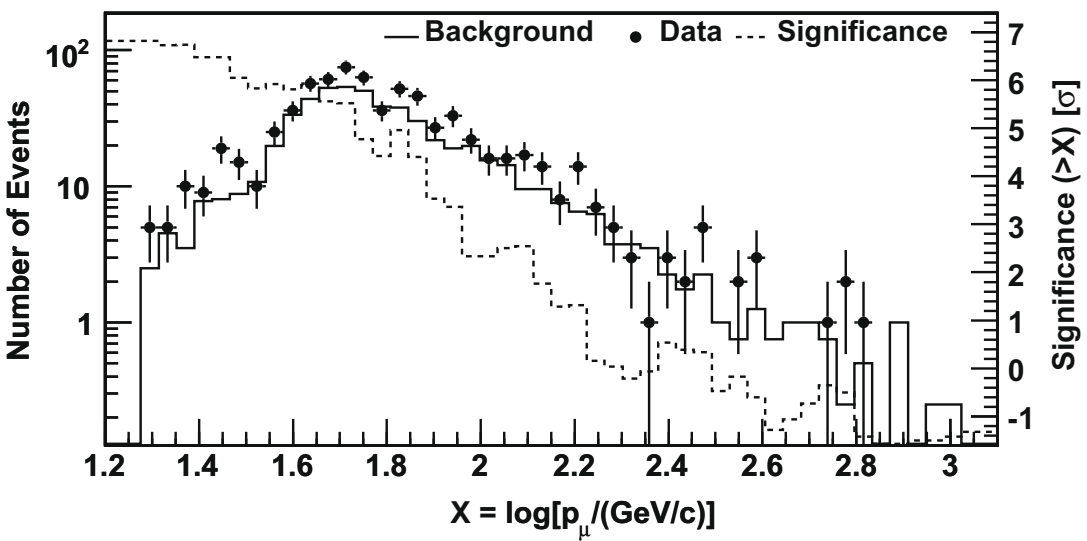

Fig. 13. Event number (data and background) as a function of the ground-level muon momentum observed in the signal cell. The integral significance is also plotted. 


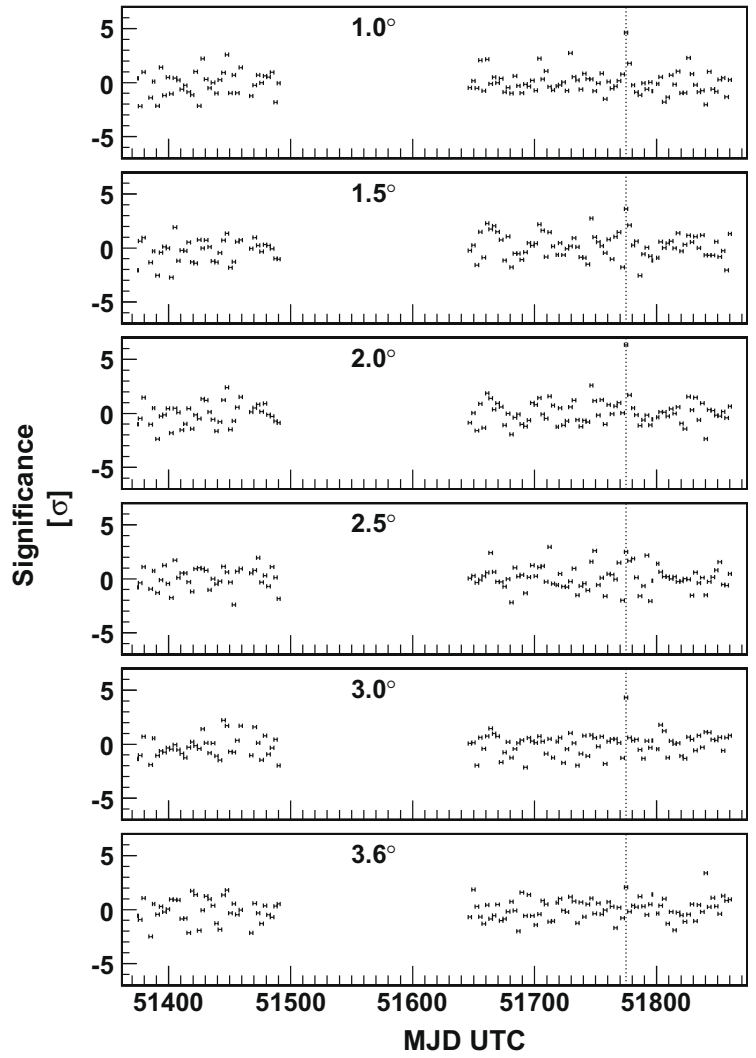

Fig. 14. The significance of the signal as a function of the chosen cell-size (time binning $\left.=2^{12} \mathrm{~min}\right)$.

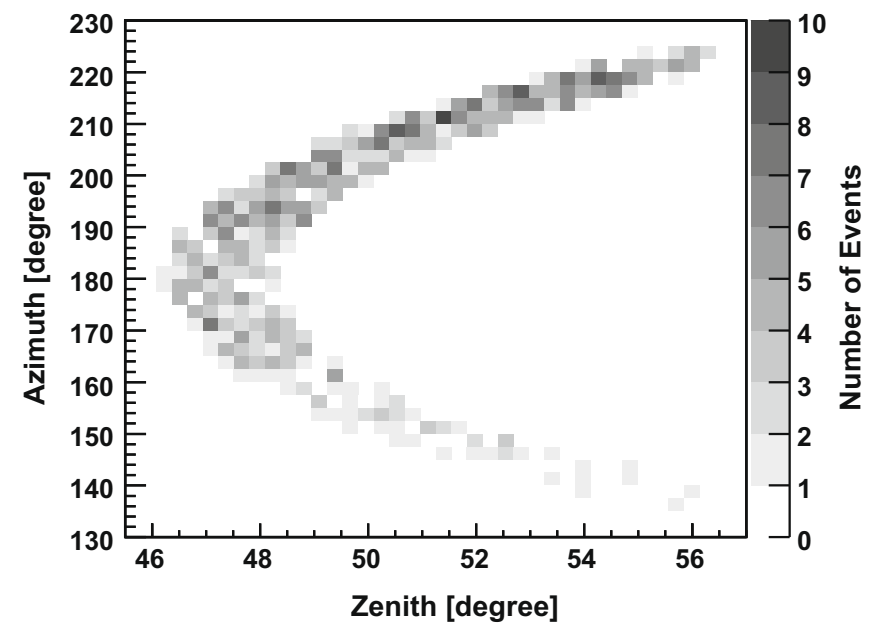

Fig. 15. Number of events collected in the signal cell as a function of azimuth and zenith angle.

cases the limit of two adjacent cells just sit at the center of the signal and therefore the significance drops in both cells.

\subsection{The low zenith angle position of the signal cell}

The fact that the sky cell emitting the signal is exposed to the detector at relatively large zenith angles and for only $20 \%$ of the time (see Fig. 11) may question the good knowledge of the acceptance. The number of events collected in this particular sky cell as a function of azimuth and zenith angle is displayed in Fig. 15. The distribution shows the asymmetric azimuth acceptance due to the shape of the detector and the expected decrease of events for large zenith angles. The fact that no particular effect shows up confirms that the extracted background estimated from the content of the neighbouring cells and this particular cell can be trusted. The excess data is also clearly visible from the event distribution as a function of the zenith- and of the azimuth-angle, Fig. 16.

In addition the expected rates agree with the calculated rates from the simulation. Fig. 17 shows the result of the acceptance calculation as defined in Chapter 2, for the particular signal cell (no cut on the muon energy is applied).

\section{Two-dimensional analysis of the excess}

In the next sections the assumption is made that the particular observed excess of events is really due to a flare signal originating from an unidentified source. To get the precise position of the signal source a two-dimensional analysis of the events around the position of the excess (from $-10^{\circ}$ to $10^{\circ}$, in declination and right ascension) is presented for the $2^{12}$ min time bin.

\subsection{Smoothing method}

First a smoothing method is used to get the contour plot of the excess. The incoming direction of every event is transformed to a new coordinate system, whose $z$-axis is pointing to the center of the excess cell, the $x$-axis points to the East, and the $y$-axis to the North. The $x-y$ plane of this new coordinate is subdivided into many pixels. For each pixel, the number of events is counted, and the number of background events of it is taken from the mean number of events in the same $y$-band in $\left[-9^{\circ},-3^{\circ}\right]$ and $\left[3^{\circ}, 9^{\circ}\right]$. Based on these numbers a new map with the same division of pixels is made, where for each pixel, the number of events $\left(N_{s}\right)$ and the number of background events $\left(N_{\mathrm{b}}\right)$ is calculated by summing up the values from the pixels in a circular area centered at the same pixel as in the previous map. The significance is then evaluated with these two numbers. Because the statistics is quite large in this case, the significance is simply estimated by $\left(N_{\mathrm{s}}-N_{\mathrm{b}}\right) / \sqrt{N_{\mathrm{b}}}$. Fig. 18 shows the map of the significances; the pixel size is selected to be $0.08^{\circ}$, the radius of the circular smoothing area is $1.08^{\circ}$, and the maximal significance is 6.742 .

\subsection{Likelihood fitting}

Secondly, a maximum likelihood method is carried out to fit the event distribution in the new coordinate system defined above. If the excess comes from a point source, the likelihood function should be

$$
\begin{gathered}
\mathscr{L}=\prod_{i}^{N} \frac{1}{w} \cdot\left(a_{0}+a_{1} y_{i}+a_{2} y_{i}^{2}\right) \\
\cdot\left[1+r \cdot \operatorname{Gauss}\left(x_{i}-x_{0}, \sigma\right) \cdot \operatorname{Gauss}\left(y_{i}-y_{0}, \sigma\right)\right], \\
\text { where } \quad w \simeq 4 a_{0} s^{2}+\frac{4}{3} a_{2} s^{4}+a_{0} r+a_{1} y_{0} r
\end{gathered}
$$

is the normalization factor, assuming that all the signal events have fallen into the fitted square region. $s$ is the half-width of the fitted square region ( $3^{\circ}$ is used), $a_{0}, a_{1}, a_{2}$ are coefficients of the polynomial fitting to the event distribution in the outer regions ( $a_{2}$ is very small). Minimizing the negative $\log$-likelihood function $-\ln \mathscr{L}$, using MINUIT, gives the following results, in Table 4:

From the fitted parameters, the following assertions about the excess can be made: 

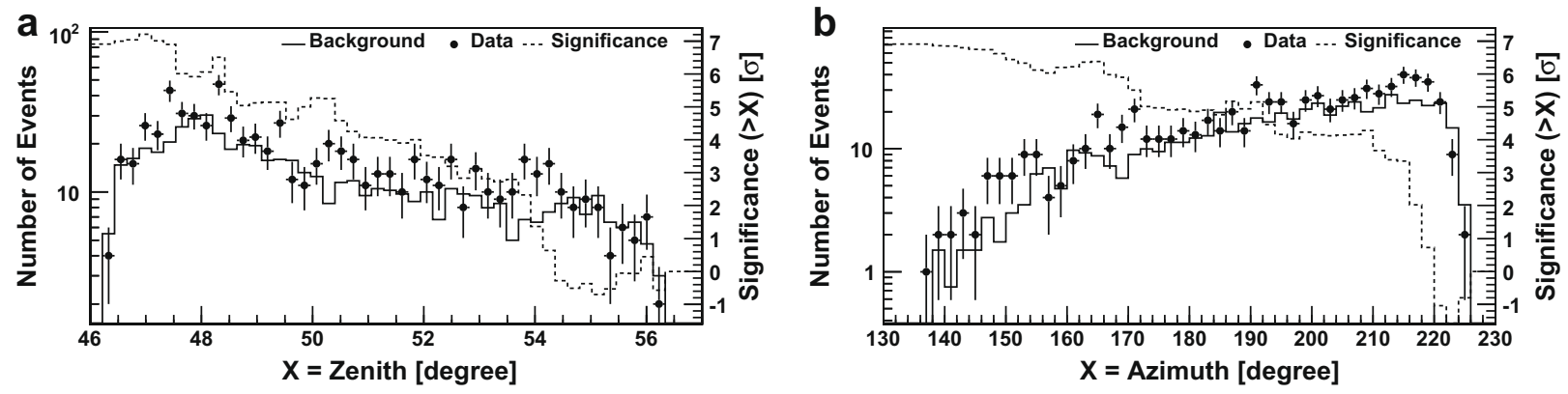

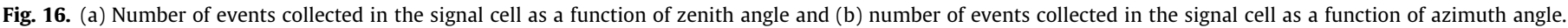

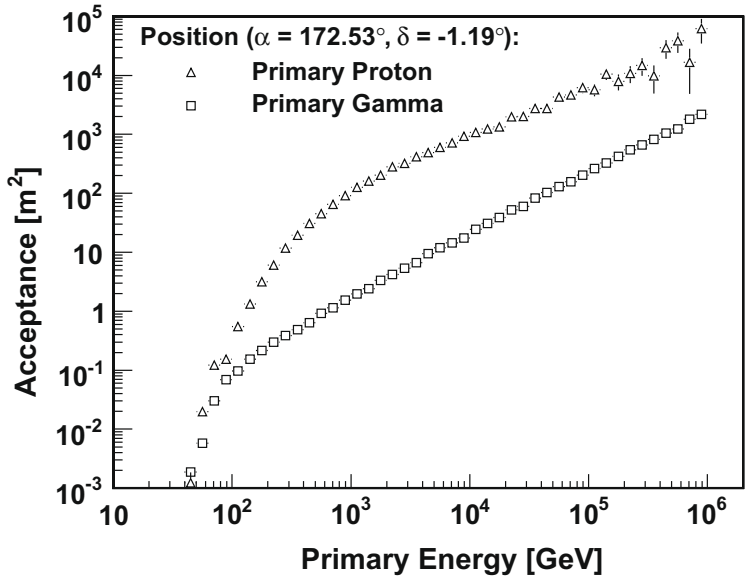

Fig. 17. The calculated acceptance for the particular signal cell for the 2.8 days of burst activity.

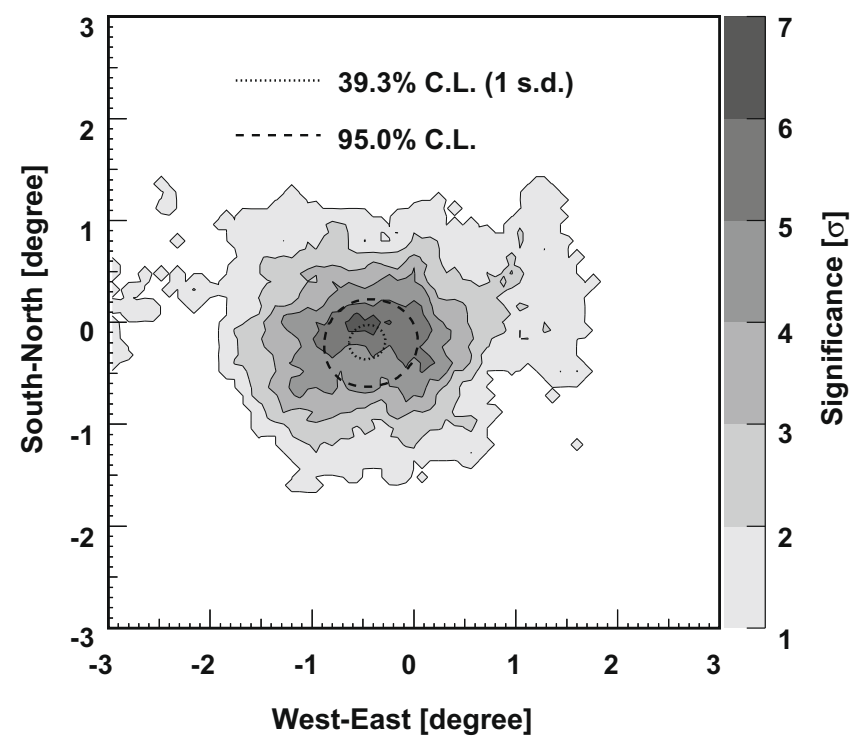

Fig. 18. The contour map of the significance, obtained by a smoothing method (see text). The center of the distribution is a fit result.

- Position: Right ascension: $\alpha=(172.53 \pm 0.17)^{\circ}=11^{\mathrm{h}} 30^{\mathrm{m}} 07.2^{\mathrm{s}}$, declination $\delta=(-1.19 \pm 0.17)^{\circ}=-1^{\circ} 11^{\prime} 24.00^{\prime \prime}$. In galactic coordinates:

longitude $=(265.02 \pm 0.42)^{\circ}$, latitude $=(55.58 \pm 0.24)^{\circ}$.

- Angular resolution: $(0.70 \pm 0.13)^{\circ}$.

- Number of total fitted excess events: $197 \pm 57$.
The fitted event distribution, including the acceptance determined asymmetric background, is shown in Figs. 19 and 20 displays the projected radial distribution of the events, as well as the fitted function. The origin of the distribution is at the fitted center of the signal. Similar studies have also been performed to demonstrate the real existence of the excess by displaying individually the signal of positive and negative muons, signals with shifted times around the medium time, muons with momenta around the average momentum, with zenith and azimuth angles slightly shifted around the mean values, etc.

The confidence levels of the position of the excess are also obtained with the MINUIT fitting, which are drawn in the previous Fig. 18. The fitted position of the excess deviates a little from the center of the contour levels, because of the larger detector acceptance towards the north being favoured by the smoothing method.

To study further the two-dimensional distribution a likelihood fit under the null hypothesis $H_{0}$ (there is no source) has been performed on the same data sample and compared to the alternative hypothesis $H_{1}$ (there is a source), as discussed above. The corresponding likelihood ratio $\chi^{2}=-2 / \ln \left(L_{0} / L_{1}\right)=31.82$ with a dof of 4. Though the chance probability that the signal is a background fluctuation equals $2.08 \times 10^{-6}$.

\subsection{The muon charge ratio}

The number of signal events with respect to the number of background events being relatively low (see Section 4 ), the deduced charge ratio $\boldsymbol{\mu}^{+} / \boldsymbol{\mu}^{-}$does not allow for a significant conclusion about the charge of the primary particles responsible for the excess. Nevertheless a study has been performed and the charge ratio as a function of the size of the cone containing the signal events, is given in Fig. 21, together with the expected ratio for primary gamma rays, protons, or nuclei according to a CORSIKA shower simulation. The (correlated) data favour a neutral primary.

\section{Flux estimation}

The estimation of the flux of gamma rays or other neutral particles emitted by this "candidate source" is strongly dependent on the assumption of the power of the primary energy spectrum and the nature of the particle. Models exist, but are the subject of debate.

An attempt to estimate the flux of gamma rays, has been made with a complete Monte Carlo simulation of the shower development in the atmosphere and the detector characteristics (see Chapter 2). Different indexes of the primary gamma energy have been assumed.

In Fig. 22 the number of simulated events below a given upper momentum threshold $p_{\mu}$ and accepted by a cone with a radius of $1.10^{\circ}$ are displayed for different indexes of the differential gamma spectra. They are normalized at infinite momentum to the also 
Table 4

The output of MINUIT: fitted parameters and errors.

\begin{tabular}{|c|c|c|c|c|c|}
\hline EXT NO. & PARAMETER NAME & VALUE & ERROR & STEP SIZE & FIRST DERIVATIVE \\
\hline 1 & Ratio(r) & $1.38524 \mathrm{e}+00$ & $3.98093 e-01$ & $7.44738 e-03$ & $1.06004 \mathrm{e}-03$ \\
\hline 2 & MeanX (xO) & $-4.64864 \mathrm{e}-\mathrm{OI}$ & $1.74791 \mathrm{e}-01$ & $1.58998 e-02$ & $-1.73802 e-03$ \\
\hline 3 & MeanY (y0) & $-1.92575 \mathrm{e}-01$ & $1.66159 \mathrm{e}-01$ & $1.56123 e-02$ & $-2.48729 e-03$ \\
\hline 4 & Sigma & $7.00141 e-01$ & $1.29050 \mathrm{e}-01$ & $8.19151 e-03$ & $2.74380 e-03$ \\
\hline
\end{tabular}

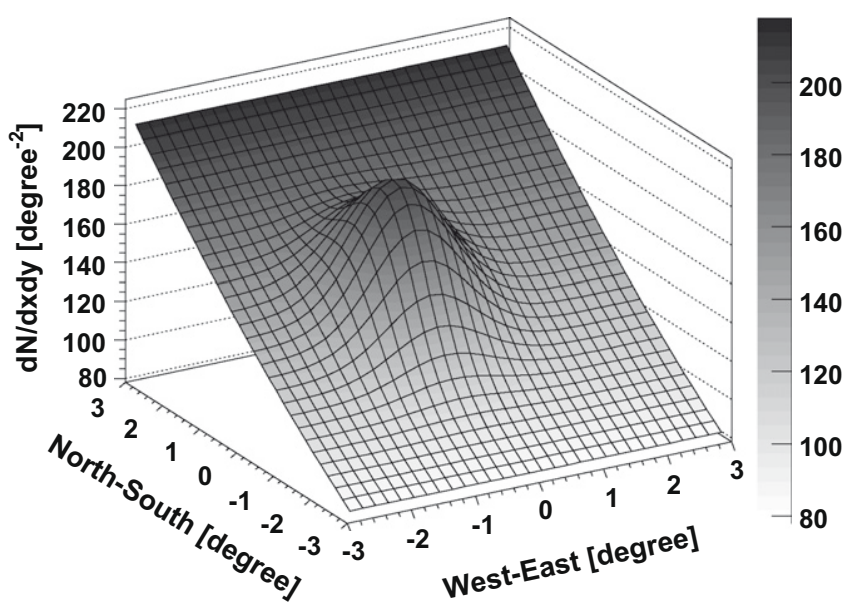

Fig. 19. The fitted event number distribution.

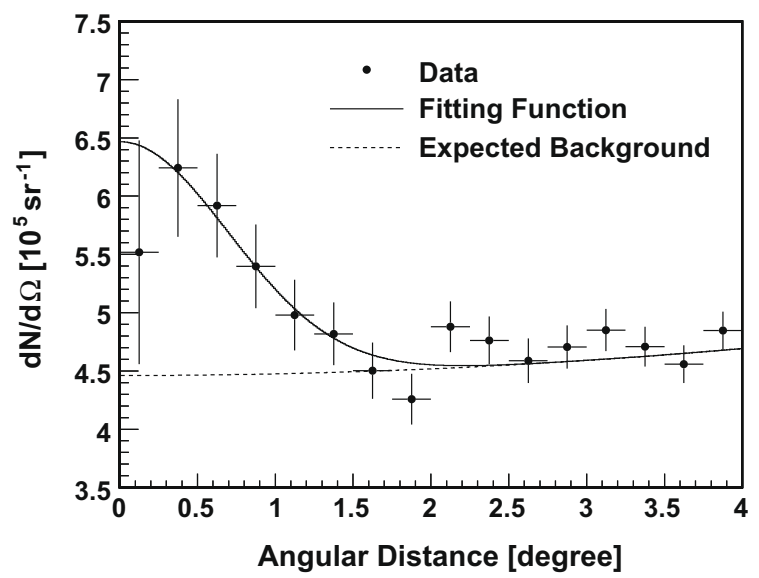

Fig. 20. Projected radial distribution of the events and the fitted function. The dashed curve shows the expected background.

displayed observed (background-) muon events with their own simulated distribution. A perfect agreement is observed, proving the good understanding of the muon background produced by primary protons and nuclei for this energy region. In addition the number of observed excess events for different muon energies below a given value is also shown.

According to the figure obtained, a rather steep spectral slope for primary gamma rays is favoured.

The likelihood (LH) fitting to the muon momentum performed to find the best slope of the gamma source delivers a differential slope of $-6.31 \pm 0.54$, see Fig. 23 . The distributions of the muon momenta for the background and the gamma sources are taken from the simulated histograms which are normalized and partly parametrized. The charge of the muons has been taken into account in this analysis. The likelihood ratio shows that the chance probability for a background fluctuation is $3.4 \times 10^{-3}$. For cone sizes different from $1.10^{\circ}$ the results are similar.

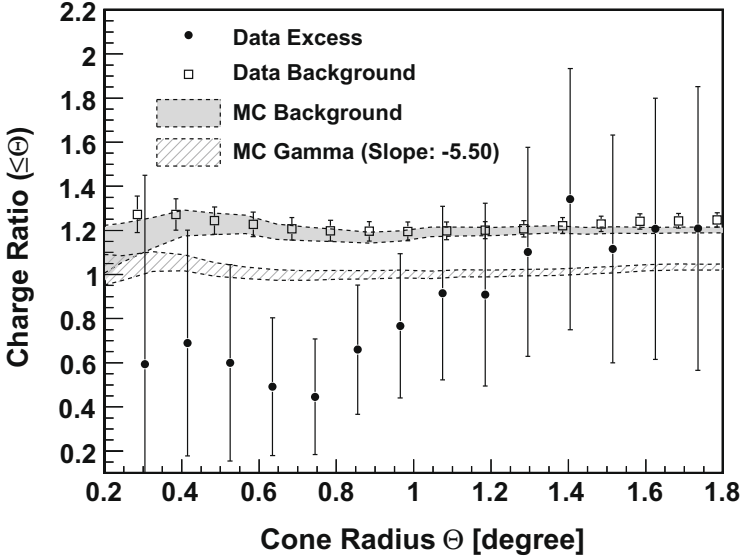

Fig. 21. Observed muon charge ratio $\mu^{+} / \mu^{-}$determined from the integral number of signal events inside a cone of given angular radius, around the center of the source position. The expected ratio for a neutral primary is given by the dashed band. The corresponding data for the muons originating from primary protons and nuclei, is also shown together with the expected ratio (gray band).

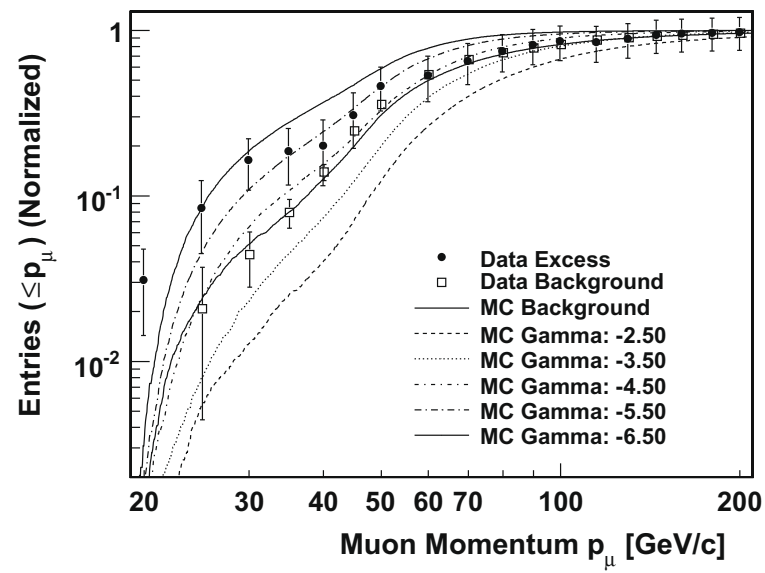

Fig. 22. Number of excess events as a function of the upper muon momentum threshold $p_{\mu}$. For comparison the MC results for spectra with different power indices of the differential primary gamma spectrum are displayed, as well as the observed and simulated muon background spectrum (solid line). All data points and curves are normalized to 1.0 at an infinite momentum threshold.

Assuming a really point like nature of the source another information about the primary spectrum is given by studying the muon angular distribution with respect to the primary's direction. This is possible due to the fact that different gamma energy-spectra will produce different angular distributions of the muons. Fig. 24 shows the angular resolution (the uncertainty of the primary's direction) as a function of the spectral slope as obtained from a Monte Carlo calculation. A linear dependence is found. The observed angular resolution of $0.70^{\circ} \pm 0.13^{\circ}$, gotten from the minimization of the negative log-likelihood function - see Section 7.2), implies therefore a slope of the differential gamma spectrum in the range of 


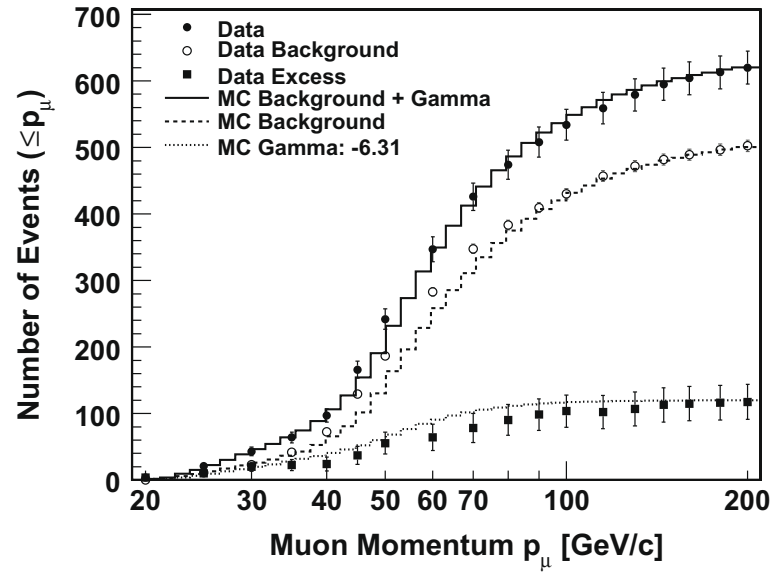

Fig. 23. The number of muon events with momenta below an upper momentum $p_{\mu}$ : shown are the data points, the sum of background data and excess events (which are also displayed individually), together with the corresponding Monte Carlo simulated distributions.

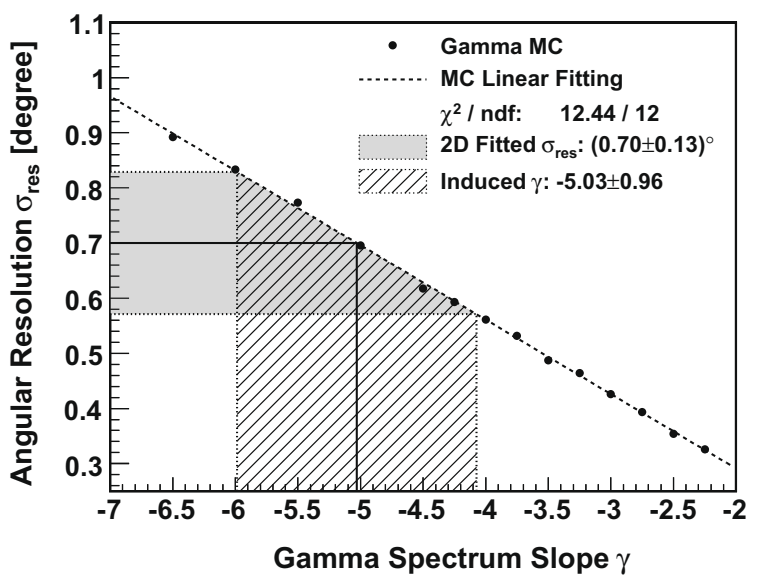

Fig. 24. Angular resolution as a function of the slope of the differential gamma spectrum.

$-5.03 \pm 0.96$, in reasonable agreement with the estimation found above.

From the above discussion a tentative differential flare spectrum could be estimated:

$\frac{d I_{\gamma}\left(E_{\gamma}\right)}{d E_{\gamma}} \approx 1.7 \times 10^{-9} \cdot E_{\gamma, \mathrm{TeV}}^{-5.5} \mathrm{~cm}^{-2} \mathrm{~s}^{-1} \mathrm{TeV}^{-1}$.

The approximation for the integral flare spectrum would then be:

$I_{\gamma}\left(>E_{\gamma}\right) \approx 4 \times 10^{-10} \cdot E_{\gamma, \mathrm{TeV}}^{-4.5} \mathrm{~cm}^{-2} \mathrm{~s}^{-1}$.
The signal data contributing between $10 \%$ and $90 \%$ to the establishment of this result are originating from primaries with rather low energies, between 41 and $140 \mathrm{GeV}$.

The expected, extrapolated flux at a threshold of $0.2,1$, resp. $10 \mathrm{TeV}$ would correspond to 2300,21 , resp. 0.023 times the corresponding Crab intensity. More details are given in Table 5.

\section{Possible source identification}

The position of the candidate flare region in the sky is illustrated in Fig. 25 and labelled "VHE L3+C" (sources presently known to emit gamma rays with energies above $100 \mathrm{GeV}$ are also shown [2]). It is located near to the supergalactic plane and may be far outside of our galaxy.

A search for "near objects" has been performed by the use of the NASA/IPAC Extragalactic Database (NED) [68]. The region shows a large density of objects. Within 10 arcmin around the VHE L3+C flare position 1350 objects (mostly galaxies) have been found, among them five IR-sources, 1 QSO, 1 QSO candidate, 1 Radio source. Within 20.4 arcmin 17 IR sources $(25 \mu \mathrm{m}), 13$ QSOs, 3 Radio sources and $1 \mathrm{X}$-ray source could be extracted from the catalogue. No intense source could be identified to be the origin of the observed flare.

No known galactic gamma source is located at the VHE $\mathrm{L} 3+\mathrm{C}$ position [2].

A search for gamma ray bursts (GRB) detected between the 14th to the 24th of August 2000 has been performed via the GRBlog [69]. Two bursts were observed without position given, and one not located at the VHE $\mathrm{L} 3+\mathrm{C}$ position.

Unfortunately no other gamma sensitive detector or satellite was running at the time of the flare reported here (17th to 20th of August 2000). The CGRO satellite reentered the atmosphere on the 4th of June 2000. Milagro was just starting data collection (the first published sky survey reports data collected between December 2000 and November 2003 for a primary energy threshold of $1 \mathrm{TeV}$ ) [70].

\section{Discussion about the flare}

According to the characteristics described in Sections 6-9, one is confronted with an unknown type of object. Most of the primaries of this unusual very steep gamma spectrum extracted from the data have an energy between 40 and $100 \mathrm{GeV}$ (not accessible to any detector running at that time). Not knowing the distance of the source, no estimation about a possible absorption due to the interaction with the interstellar matter can be made.

Gamma ray bursts with $\mathrm{GeV}$ gamma ray emission exist (e.g. [72]), but according to our knowledge none emitting high energy signals for at least three days.

The starburst galaxy NGC 253 [5] exhibits a spectrum with an index of -3.74 (suggesting a cutoff). This is already a quite

Table 5

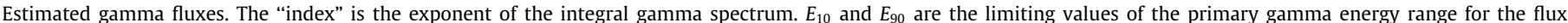

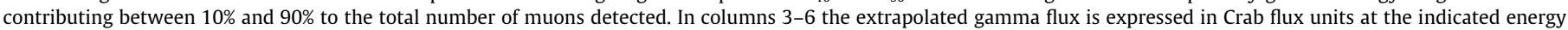

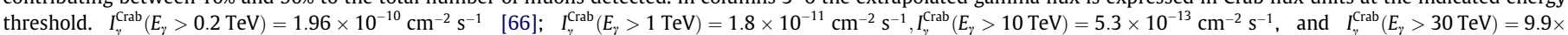
$10^{-14} \mathrm{~cm}^{-2} \mathrm{~s}^{-1}$ [67].

\begin{tabular}{|c|c|c|c|c|c|c|}
\hline Index & $E_{10}(\mathrm{GeV})$ & $E_{90}(\mathrm{GeV})$ & Flux $>0.2 \mathrm{TeV}(\mathrm{Crab})$ & Flux $>1 \mathrm{TeV}(\mathrm{Crab})$ & Flux $>10 \mathrm{TeV}$ (Crab) & Flux $>30 \mathrm{TeV}(\mathrm{Crab})$ \\
\hline-2.0 & 79 & $1.3 \times 10^{3}$ & $2.0 \times 10^{4}$ & $1.1 \times 10^{4}$ & $3.6 \times 10^{3}$ & $2.1 \times 10^{3}$ \\
\hline-2.5 & 68 & $4.9 \times 10^{2}$ & $1.6 \times 10^{4}$ & $3.9 \times 10^{3}$ & $4.1 \times 10^{2}$ & $1.4 \times 10^{2}$ \\
\hline-3.5 & 51 & $2.1 \times 10^{2}$ & $7.3 \times 10^{3}$ & $3.4 \times 10^{2}$ & $3.6 \times 10^{0}$ & $4.2 \times 10^{-1}$ \\
\hline-4.5 & 41 & $1.4 \times 10^{2}$ & $2.3 \times 10^{3}$ & $2.1 \times 10^{1}$ & $2.3 \times 10^{-2}$ & $8.6 \times 10^{-4}$ \\
\hline-5.0 & 38 & $1.2 \times 10^{2}$ & $1.2 \times 10^{3}$ & $5.1 \times 10^{0}$ & $1.7 \times 10^{-3}$ & $3.9 \times 10^{-5}$ \\
\hline-5.5 & 36 & $1.1 \times 10^{2}$ & $6.3 \times 10^{2}$ & $1.2 \times 10^{0}$ & $1.3 \times 10^{-4}$ & $1.6 \times 10^{-6}$ \\
\hline
\end{tabular}




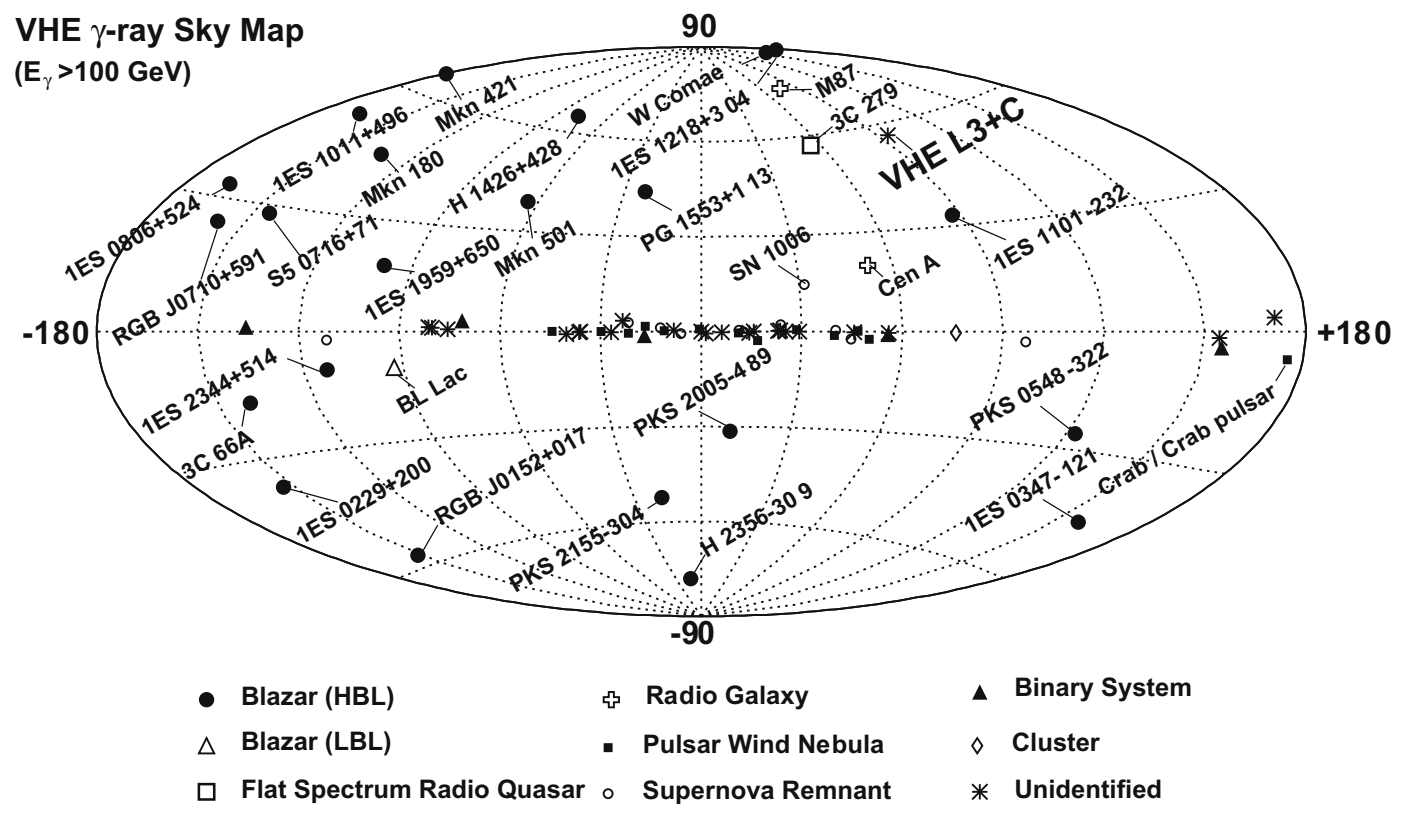

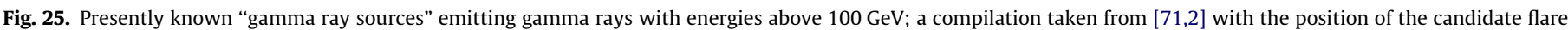

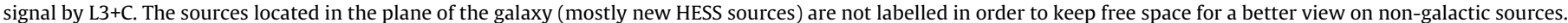

exceptionally steep spectrum, but it represents still a flatter one than the estimated spectrum of the VHE L3+C flare.

An AGN would be a more realistic candidate source, since flux variations of this order have been observed by the HEGRA-, or the HESS-collaborations and others for higher energy gamma rays. But all AGN or blazar spectra are much flatter than the one of the VHE L3+C object. Extrapolations from the reported flux in Section 8 between 40 and $140 \mathrm{GeV}$ to $0.8-10 \mathrm{TeV}$ give comparable flux values to Mrk 421 or Mkn 501 at these higher energies [73].

M87 may have emitted a flare with comparable time dependence, but observed at higher energies [6]. With respect to the low number of events observed from VHE L3+C no attempt has been made to search for short time bursts inside of the three days lasting flare, as was possible for the M87 bursts [6]. Ignoring a Doppler-factor the diameter $D$ of the signal emitting region of the observed VHE L3+C flare having lasted for three days would amount to some $8 \times 10^{13} \mathrm{~m}$. Assuming further $D$ being the Schwarzschild diameter of a central black hole $(\mathrm{BH})$, the mass of this $\mathrm{BH}$ would be equal to $1.3 \times 10^{10} M_{\odot} \approx 4.5 \times M_{M 87}$. The steep energy spectrum given in Section 8 and integrated between 41 and $140 \mathrm{GeV}$ (the energy range inside which most of the signal has been recorded) gives a fluence of $15 \mathrm{erg} \mathrm{cm}^{-2}$ emitted in three days. From an extrapolation to an energy range between $730 \mathrm{GeV}$ and $10 \mathrm{TeV}$ one gets a fluence of $6 \times 10^{-4} \mathrm{erg} \mathrm{cm}^{-2}$, a factor 600 larger than the fluence of the March 2005 flare from M87.

For an arbitrary distance of the source of $75 \mathrm{Mpc}$ the total energy emitted isotropically into $4 \pi$ corresponds roughly to $10^{55} \mathrm{erg}$. This may be compared to GRB energies of $10^{52}$ to $10^{54} \mathrm{erg}$.

In summary, no known source exhibits the characteristics of the VHE L3+C signal.

\section{Conclusions}

An unusual very intense flare of very high energy and most probably of neutral nature cosmic rays has been detected with the underground muon spectrometer $\mathrm{L} 3+\mathrm{C}$ at CERN. The burst was detected with a chance probability of $2.6 \times 10^{-3}$ in a particular sky cell, through muons produced in the atmosphere. The signal originating from primaries with energies below a TeV, exhibits a clear time evolution over three days, persists up to muon energies above $50 \mathrm{GeV}$ and was recorded within opening angles of $2.5^{\circ}$ down to less than $1^{\circ}$, contrary to what is expected from a purely statistical fluctuation. It happened between 51773.489 and 51776.333 MJD (2.8 days). The candidate source is located at a galactic longitude of $(265.02 \pm 0.42)^{\circ}$ and latitude of $(55.58 \pm$ $0.24)^{\circ}$.

\section{Acknowledgements}

The $\mathrm{L} 3+\mathrm{C}$ collaboration would like to thank CERN for the support given to this experiment (RE 4) and express in particular its gratitude to the IT division taking care of the $\mathrm{L} 3+\mathrm{C}$ computer farm, as well as to the crew operating at LEP point 2, having delivered a big effort for the installation of the additional hardware. A special thank is addressed to our L3 colleagues for their help during the data taking periods.

\section{References}

[1] D. Horan, T.C. Weekes, New Astron. Rev. 48 (2004) 527; W. Hoffmann et al., HESS collaboration, in: Proceedings of the XXIXth ICRC, Pune, India, 2005, pp. 10-97.;

R.A. Ong, Phys. Rep. 305 (1998) 93, in: Proceedings of the XXIXth ICRC, Pune, India, 2005, pp. 10-329.;

P.L. Bierman et al., Astron. Astrophys. 369 (2000) 269.

[2] R. Wagner, <http://www.mppmu.mpg.de/rwagner/sources/>.

[3] J. Hinton, arXiv : 0712.3352v1[astro - ph]20Dec.2007, in: Proceedings of the XXXth ICRC 30th ICRC, Merida, Mexico, 2007.

[4] F. Aharonian et al., HESS collaboration, Astron. Astrophys. 460 (2006) 743.

[5] C. Itoh et al., Astron. Astrophys. 396 (2002) L1-L4.

[6] F. Aharonian et al., HESS collaboration, Science 314 (2006) 1424.

[7] F. Aharonian et al., HEGRA collaboration, Astron. Astrophys. 393 (2002) L37; F. Aharonian et al., HESS collaboration, Science 307 (2005) 1938.

[8] R.C. Hartman et al., Astrophys. J. Suppl. 123 (1) (1999) 79 (3rd EGRET catalogue of High-Energy Gamma-Ray Sources).

[9] J. Clear et al., COS-B collaboration, Astron. Astrophys. 174 (1987) 85.

[10] M.F. Cawley et al., Whipple collaboration, Exp. Astron. 1 (1990) 173.

[11] L. Resvanis et al., Haleakala collaboration, in: Proceedings of the NATO Advanced Research Workshop, Durham, England, August 11-15, 1986. Dodrecht, D. Reidel Publ. Co., 1987, p. 131.

[12] A. Barrau et al., CAT collaboration, NIM A 416 (1998) 278.

[13] M. De Naurois et al., CELESTE collaboration, Astrophys. J. 566 (2002) 343.

[14] F. Aharonian et al., AIROBICC collaboration, Astron. Astrophys. 327 (1997) L5 K. Okumura et al., AIROBICC collaboration, Astrophys. J. Lett. 579 (2002) L9L12;

T. Antoni et al., AIROBICC collaboration, Astrophys. J. 608 (2004) 865 
[15] L.M. Boone et al., STACEE collaboration, Astrophys. J. 579 (2002) L5.

[16] Yu.I. Neshpor et al., Astron. Lett. 24 (1998) 134

[17] T. Tanimori et al., ApJ 492 (1998) L33.

[18] A. Alkofer et al., in: Proceedings of the 18th ICRC, Bangalore, 1983.; B.L. Dingus et al., Phys. Rev. Lett. 60 (1988) 1785.

[19] M. Amenomori et al., Tibet collaboration, Astrophys. J. 532 (2000) 302.

[20] A. Daum et al., HEGRA collaboration, Astropart. Phys. 8 (1997) 1.

[21] B. Khélifi, et al., HESS collaboration, in: Proceedings of the XXXth ICRC, Merida, 2007, p. 986.

[22] D. Berge et al., HESS collaboration, in: Proceedings of the XXXth ICRC, Merida, 2007, p. 524.

[23] O.C. de Jager, F.W. Stecker, Astrophys. J. 566 (2002) 738.

[24] F. Aharonian et al., Nature 440 (2006) 1018.

[25] N. Bhatt et al., TACTIC collaboration, in: Proceedings of the XXVIIth ICRC Hamburg, 2001, p. 2391.;

K.K. Yadav, Nucl. Inst. Meth. A 527 (2002) 411.

[26] T. Hara et al., CANGAROO collaboration, Nucl. Inst. Meth. A 332 (1993) 300.

[27] J.A. Hinton, HESS collaboration, New Astron. Rev. 48 (2004) 331.

[28] E. Lorenz, MAGIC collaboration, New Astron. Rev. 48 (2004) 339.

[29] J. Holder et al., VERITAS collaboration, Astropart. Phys. 25 (2006) 391; J. Quinn et al., VERITAS collaboration, in: Proceedings of the XXVIIth ICRC, Hamburg, 2001, p. 2781.

[30] A.N. Otte et al., MAGIC collaboration, in: Proceedings of the XXXth ICRC, Merida, 2007, p. 1078

[31] A. Aloisio et al., ARGO collaboration, Il Nuovo Cim. 24C (2001) 739; R. Assiro et al., ARGO collaboration, in: Proceedings of the XXVIIth ICRC Hamburg, 2001, p. 2883.

[32] P.K. Mohanty et al., in: Proceedings of the XXIXth ICRC, Pune, India, 2005, pp. 6-21.;

S.K. Gupta et al., GRAPES collaboration, Phys. Rev. D 68 (2003) 052005.

[33] G. Sullivan et al., MILAGRO collaboration, in: Proceedings of the XXVIIth ICRC, Hamburg, 2001.;

R. Atkins et al., Nucl. Instr. Meth. A 499 (2000) 478;

R. Atkins et al., Astrophys. J. Lett. 533 (2000) L119;

R. Atkins et al., Astrophys. J. Lett. 525 (1999) L25.

[34] A.A. Abdo et al., Astrophys. J. 664 (part 2) (2007) L000; A.A. Abdo, Thesis, Michigan State University, 2007.

[35] O. Adriani et al., L3+C collaboration, Nucl. Inst. Meth. A 488 (2002) 209.

[36] P. Achard et al., L3 collaboration, Phys. Lett. B 598 (2004) 15.

[37] F. Halzen, T. Stanev, B. Yodh, Phys. Rev. D55 (1997) 4475; T. Stanev, Phys. Rev. D33 (1986) 2740.

[38] M. Drees, F. Halzen, K. Hikasa, Phys. Rev. D39 (1989) 1310.

[39] J. Albert et al., MAGIC collaboration, ApJ 669 (2007) 862; J. Albert et al., MAGIC collaboration, Phys. Lett. B 668 (2008) 253.

[40] F. Aharonian et al., HESS collaboration, ApJ Lett. 664 (2007) L71.

[41] S.P. Ahlen et al., MACRO collaboration, Astrophys. J. 412 (1993) 301; C. Satriano et al., MACRO collaboration, in: Proceedings of the XXVI ICRC, Salt Lake City, 1999.

[42] G. Battistoni et al., NUSEX collaboration, Phys. Lett. B155 (1985) 465

[43] M. Ambrosio et al., MACRO collaboration, Astropart. Phys. 18 (2003) 615

[44] M.L. Marshak et al., Phys. Rev. Lett. 54 (1985) 2079; M.L. Marshak et al., Phys. Rev. Lett. 55 (1985) 1965; M.A. Thomson et al., Phys. Lett. B269 (1991) 220.
[45] R. Bionta et al., IMB collaboration, Phys. Rev. D36 (1987) 30; J.A. Kochocki et al., SOUDAN 2 collaboration, Phys. Rev. D42 (1990) 2967; Y. Oyama et al., Kamiokande collaboration, Phys. Rev. Lett. 56 (1986) 991; Homestake collaboration, Astropart. Phys. 18 (2003) 615.

Ch. Berger et al., Fréjus collaboration, Phys. Lett. B171 (1986) 118.

[46] A. Borione et al., CASA-MIA collaboration, Phys. Rev. D 55 (1997) 1714.

[47] CYGNUS collaboration, Phys. Rev. Lett. 60 (1988) 1785.

[48] T. Antoni et al., KASCADE collaboration, Astrophys. J. 608 (2004) 865.

[49] M. Sasano et al., Ooty experiment, in: Proceedings of the ICRC, Salt Lake city, OG 2.4.14, 1999.;

S.C. Tonwar, Bull. Ind. Astron. Soc. India 30 (2002) 147.

[50] P. Achard et al., L3 collaboration, Astropart. Phys. 25 (2006) 298.

[51] R. Ramelli, Search for Cosmic Ray Point Sources and Anisotropy Measurement with the L3+C Experiment, Ph.D. Thesis No. 14683, ETH-Zürich, 2002.

[52] B. Adeva et al., L3 collaboration, Nucl. Instr. Meth. A 289 (1990) 35.

[53] P. Achard et al., L3 collaboration, Astropart. Phys. 23 (2005) 411.

[54] J.-F. Parriaud, Recherche d'antimatière dans les rayons cosmiques en utilisant l'ombre de la lune et de sources cosmiques avec le détecteur à muons de L3. Ph.D. Thesis, Université Claude Bernard, Lyon I, 2003.

[55] B. Weibel-Sooth, P.L. Biermann, H. Meyer, Astron. Astrophys. 330 (1998) 389398.

[56] D. Heck et al., Technical Report, FZKA 6019, Forschungszentrum Karlsruhe, 1998.

[57] N.N. Kalmykov, S.S. Ostapchenko, A.I. Pavlov, Nucl. Phys. B (Suppl. 52) (1997) 17.

[58] R. Brun et al., GEANT 3, CERN Report DD/EE/84-1, 1987.

[59] T. Antoni et al., Astrophys. J. 608 (2004) 865.

[60] R. Atkins et al., Astrophys. J. 595 (2003) 803.

[61] D.E. Alexandreas et al., Nucl. Instr. Meth. A 328 (1993) 570.

[62] T.P. Li, Y.Q. Ma, Astrophys. J. 272 (1983) 317.

[63] S. Wilks, Mathematical Statistics, Wiley, New York, 1962 (Chapter 13.8).

[64] W. Eadie et al., Statistic Methods in Experimental Physics, North-Holland, Amsterdam, 1971 (Chapter 10.5.2)

[65] T.P. Li, Mathematical Treatment in Experiments, Science Press, Beijing, 1980 (Chapter 6.3.4).

[66] J. Albert et al., MAGIC collaboration, Astrophys. J. 674 (2008) 1037.

[67] T. Tanimori et al., CANGAROO collaboration, Astrophys. J. 492 (1998) L33.

[68] NASA/IPAC Extragalactic Database (NED), Operated by the Jet Propulsion Laboratory, California Institute of Technology, Under Contract with the National Aeronautics and Space Administration. <http://nedwww.ipac. caltech.edu/>.

[69] <http://grad40.as.utexas.edu/grblog.php>.

[70] R. Atkins et al., Milagro collaboration, Astrophys. J. 608 (2004) 680

[71] D. Horan, T.C. Weekes, New Astron. Rev. 48 (2004) 527.

[72] R. Atkins et al., Astrophys. J. 583 (2003) 824.

[73] M. Schubnell et al., in: Proceedings of the VIIth International Symposium on Very High Energy Cosmic Ray Interactions AIP Conference, 276 (1993) 185. J.A. Gaidos et al., Nature 383 (1996) 26; F. Krennrich et al., in: Proceedings of the XXVI ICRC, Salt Lake City, 1999, in: Proceedings of the XXV ICRC, Durban 1997.;

F.A. Aharonian et al., Astron. Astrophys. 349 (1999) 11;

K. Okumura et al., Astrophys. J. Lett. 579 (2002) L9-L12;

F.A. Aharonian et al., Astron. Astrophys. 410 (2003) 813 